\title{
Incoherent pion photoproduction on the deuteron with polarization observables II: Influence of final state rescattering
}

\author{
A. Fix and H. Arenhövel \\ Institut für Kernphysik, Johannes Gutenberg-Universität Mainz, D-55099 Mainz, Germany
}

(Dated: March 29, 2018)

\begin{abstract}
Incoherent pion photoproduction on the deuteron is studied for photon energies from threshold up to $1 \mathrm{GeV}$ with special emphasis on polarization observables. The elementary $\gamma N \rightarrow \pi N$ amplitude is taken from the MAID model. The influence of final state interactions on total and semi-exclusive cross sections $\vec{d}(\vec{\gamma}, \pi) N N$ is investigated by including complete rescattering in the final $N N$ - and $\pi N$-subsystems. For charged pion-production the influence of $N N$-rescattering is moderate whereas $\pi N$-rescattering is almost negligible. Much stronger influences of $N N$-rescattering are seen in neutral pion production, which is due to the elimination of a significant spurious coherent contribution in the impulse approximation. Sizeable effects are also found in some of the beam, target and beam-target asymmetries of the differential cross section.
\end{abstract}

PACS numbers: 13.60.Le, 21.45.+v, 24.70.+s, 25.20.Lj

\section{INTRODUCTION}

Photoproduction of pions on the deuteron has two main but complementary points of interest. The first one is to obtain information on the elementary reaction on the neutron by using the deuteron as an effective neutron target. A prerequisite for this is that one has reliable control on off-shell and medium effects. In order to minimize such effects, quasi-free kinematics is preferred. The second but not secondary aspect is just the influence of a nuclear environment on the production process, for the study of which off-quasi-free kinematics is better suited.

Pion photoproduction on the deuteron has been studied quite extensively over the past 50 years, starting with early work in [1, 2, 3]. The role of final state interaction (FSI) has been investigated by Laget [4, 5] applying a diagrammatic approach. The influence of FSI effects were found to be quite small for charged pion photoproduction compared to the neutral channel. A satisfactory agreement with experimental data was achieved for $\pi^{-}$production [6]. Subsequently, these results were confirmed by Levchuk et al. [7] for the $d\left(\gamma, \pi^{0}\right) n p$ reaction for which the elementary photoproduction operator of Blomqvist and Laget [3] was used. This work was improved and extended to charged pion production channels in 8], where a more realistic elementary production operator from the SAID 99 and MAID [10] multipole analyses was taken and $N N$-rescattering included, based on the Bonn r-space potential 11. The influence of $N N$-FSI was confirmed and good agreement with experimental data was achieved. In the threshold region a sizeable effect from $\pi N$-rescattering was noted in [12] which arises from intermediate charged pion production with subsequent charge exchange rescattering on the spectator nucleon. The influence of $N N$ - and $\pi N$-rescattering on polarization observables has been investigated in [7] for the GDH sum rule in the $\pi^{0} n p$-channel as well as in [13] for target asymmetries in the $\pi^{-} p p$-reaction.

FSI effects in incoherent pion photoproduction were also studied by Darwish et al. 14]. The same approach was then applied to the spin asymmetry with respect to circularly polarized photons and vector polarized deuterons [15], which determines the much discussed Gerasimov-Drell-Hearn sum rule [16]. However, the approach was limited to the $\Delta(1232)$-resonance region in view of a relatively simple elementary production operator, based on an effective Lagrangian approach from Schmidt et al. 17]. A puzzling result of this work was that the influence of FSI on the total cross sections for charged pion production resulted in a slight decrease in the $\Delta$-resonance region in contrast to previous work [4, 5, 8] where a slight increase was found. Recently, this work was extended in a series of papers [18, 19, 20, 21, 22. to a study of various polarization asymmetries of the semi-exclusive differential cross section for $\vec{d}(\vec{\gamma}, \pi) N N$. The semiexclusive beam asymmetry $\Sigma$ for linearly polarized photons and the target asymmetries $T_{I M}$ with respect to polarized deuterons were considered in [18] and beam-target asymmetries in 19], in both cases only in impulse approximation. Final state interaction effects were subsequently discussed in 20, 21, 22]. Unfortunately, many of these results are based on incorrect expressions for polarization observables as pointed out recently in 23], where a thorough derivation of these observables is given.

Thus the present work was motivated firstly to use a better elementary production operator from the MAID model 10], allowing one to go to higher photon energies and also to give a more reliable description of the threshold region. Secondly, we would like to clarify the role of the final state interaction (FSI) in view of the above mentioned differences in the role of FSI effects. Thirdly, the increasing importance of polarization observables requires a more thorough and reliable treatment as done in [18, 19, 20, 21, 22]. In the present work we consider again besides 
the impulse approximation (IA) complete rescattering in the final two-body subsystems, i.e. in the $N N$ - and $\pi N$ subsystems. Results on the spin asymmetry of the total cross section, based on the present approach, and its contribution to the Gerasimov-Drell-Hearn sum rule have already been reported in 24].

In the next section we briefly review the basic formalism for the general differential cross section with inclusion of polarization observables as derived in [23]. Furthermore, the essential ingredients for the calculation of the $T$ matrix in the impulse approximation (IA) and the rescattering contributions are described here. The results on the unpolarized differential cross section for the semi-exclusive process $\vec{d}(\vec{\gamma}, \pi) N N$ as well as all beam, target, and beamtarget asymmetries will be presented and discussed in Sect. III together with a comparison to existing data. Finally, we will conclude in Sect. IV with a summary and an outlook. The separation of the the various asymmetries of the semi-exclusive differential cross section are discussed in Appendix $\mathrm{A}$, and a modified impulse approximation is given in Appendix B]

\section{THE FORMALISM}

To begin with, we will briefly outline the kinematic framework of the reaction under study, namely

$$
\gamma\left(k, \vec{\varepsilon}_{\mu}\right)+d\left(p_{d}\right) \rightarrow \pi(q)+N_{1}\left(p_{1}\right)+N_{2}\left(p_{2}\right),
$$

defining the notation of the four-momenta of the participating particles. The circular polarization of the photon is denoted by $\vec{\varepsilon}_{\mu}(\mu= \pm 1)$. For the description of cross sections and polarization observables we take as reference frame the laboratory frame and as independent variables for the characterization of the final state the outgoing pion momentum $\vec{q}=\left(q, \theta_{q}, \phi_{q}\right)$ and the spherical angles $\Omega_{p}=\left(\theta_{p}, \phi_{p}\right)$ of the relative momentum $\vec{p}=\left(\vec{p}_{1}-\vec{p}_{2}\right) / 2=\left(p, \Omega_{p}\right)$ of the two outgoing nucleons. The coordinate system is chosen as right-handed with $z$-axis along the photon momentum $\vec{k}$. According to the convention of [23], we distinguish in general three planes: (i) the photon plane spanned by the photon momentum and the direction of maximal linear photon polarization, which defines the direction of the $x$-axis, (ii) the pion plane, spanned by photon and pion momenta, which intersects the photon plane along the $z$-axis with an angle $\phi_{q}$, and (iii) the nucleon plane spanned by total and relative momenta of the two nucleons. It intersects the pion plane along the total momentum of the two nucleons (see Fig. 1). In case the linear photon polarization vanishes, one can choose $\phi_{q}=0$ and then photon and pion planes coincide.

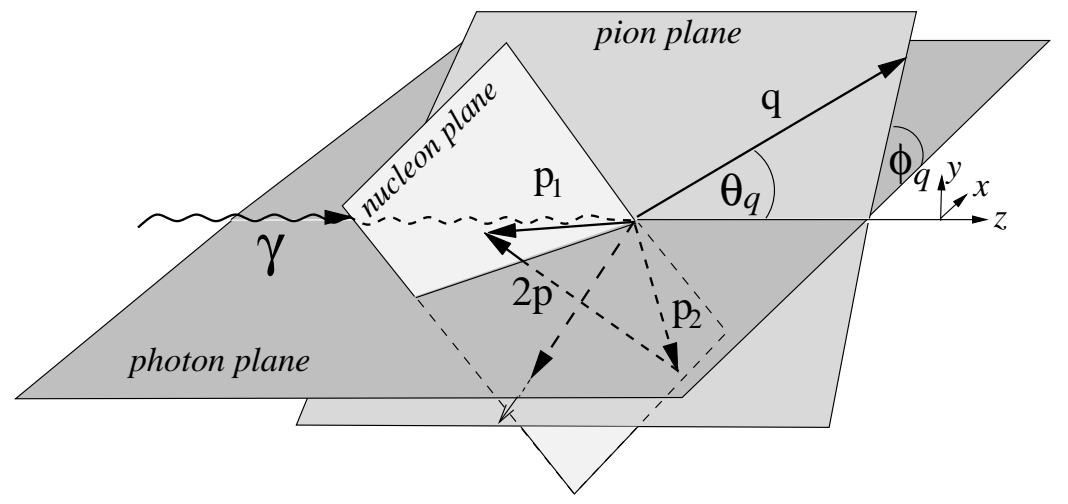

FIG. 1: Kinematics of pion photoproduction on the deuteron in the laboratory system.

\section{A. The $T$-matrix}

All observables are determined by the $T$-matrix elements of the electromagnetic pion production current $J_{\gamma \pi}$ between the initial deuteron and the final $\pi N N$ states

$$
T_{s m_{s}, \mu m_{d}}\left(q, \Omega_{q}, \Omega_{p}\right)=-{ }^{(-)}\left\langle\vec{q}, \vec{p} ; s m_{s}\left|\vec{\varepsilon}_{\mu} \cdot \vec{J}_{\gamma \pi}(0)\right| \vec{d} ; 1 m_{d}\right\rangle
$$

where $s$ and $m_{s}$ denote the total spin and its projection on the relative momentum of the outgoing two nucleons and $m_{d}$ correspondingly the deuteron spin projection on the chosen $z$-axis. As is shown in [23], the dependence on $\phi_{q}$ can be split of, i.e.

$$
T_{s m_{s} \mu m_{d}}\left(q, \Omega_{q}, \Omega_{p}\right)=e^{i\left(\mu+m_{d}-m_{s}\right) \phi_{q}} t_{s m_{s} \mu m_{d}}\left(q, \theta_{q}, \theta_{p}, \phi_{p q}\right)
$$


Thus the small $t$-matrix depends besides on $q, \theta_{q}$, and $\theta_{p}$ only on the difference of the azimuthal angles of $\vec{q}$ and $\vec{p}$, i.e. on $\phi_{p q}=\phi_{p}-\phi_{q}$. The small $t$-matrix elements are the basic quantities which determine the differential cross section and asymmetries. The latter are listed explicitly in [23].

For the calculation of the $T$-matrix we start from the impulse approximation (IA) to which the contributions from $N N$ - and $\pi N$-rescattering are added. Possible two-body contributions to the electromagnetic interaction are neglected. Thus the treatment is completely analogous to previous work on incoherent $\pi$ - and $\eta$-photoproduction on the deuteron [14, 25] to which the reader is referred for formal details. Then the $T$-matrix is given by the sum

$$
T_{s m_{s} \mu m_{d}}=T_{s m_{s} \mu m_{d}}^{I A}+T_{s m_{s} \mu m_{d}}^{N N}+T_{s m_{s} \mu m_{d}}^{\pi N} .
$$

For the IA contribution, which describes the production on one nucleon while the other acts as a spectator, one has

$$
\begin{aligned}
T_{s m_{s} \mu m_{d}}^{I A} & =\left\langle\vec{q}, \vec{p}, s m_{s}\left|\left[t_{\gamma \pi}(1)+t_{\gamma \pi}(2)\right]\right| 1 m_{d}\right\rangle \\
& =\sum_{m_{s}^{\prime}}\left(\left\langle s m_{s}\left|\left\langle\vec{p}_{1}\left|t_{\gamma \pi}\left(W_{\gamma N_{1}}\right)\right|-\vec{p}_{2}\right\rangle \phi_{m_{s}^{\prime} m_{d}}\left(\vec{p}_{2}\right)\right| 1 m_{s}^{\prime}\right\rangle-(1 \leftrightarrow 2)\right),
\end{aligned}
$$

where $t_{\gamma \pi}$ denotes the elementary pion photoproduction operator, which we take from the MAID model, $W_{\gamma N_{1}}$ the invariant energy of the $\gamma N$ system, and $\vec{p}_{1 / 2}=(\vec{k}-\vec{q}) / 2 \pm \vec{p}$, and $\phi_{m_{s} m_{d}}(\vec{p})$ is related to the internal deuteron wave function in momentum space by

$$
\left\langle\vec{p}, 1 m_{s} \mid 1 m_{d}\right\rangle^{(d)}=\phi_{m_{s} m_{d}}(\vec{p})=\sum_{L=0,2} \sum_{m_{L}} i^{L}\left(L m_{L} 1 m_{s} \mid 1 m_{d}\right) u_{L}(p) Y_{L m_{L}}(\hat{p}) .
$$

In view of the fact that for neutral pion production the major influence of $N N$-rescattering arises from the nonorthogonality of the final $N N$-plane wave to the deuteron ground state, we have considered in addition a modified IA-amplitude, for which the deuteron component is projected out from the final plane wave (see Appendix B for details).

The two rescattering contributions have a similar structure

$$
\begin{aligned}
T_{s m_{s} \mu m_{d}}^{N N} & =\left\langle\vec{q}, \vec{p}, s m_{s}\left|t_{N N} G_{N N}\left[t_{\gamma \pi}\left(W_{\gamma N_{1}}\right)+t_{\gamma \pi}\left(W_{\gamma N_{2}}\right)\right]\right| 1 m_{d}\right\rangle, \\
T_{s m_{s} \mu m_{d}}^{\pi N} & =\left\langle\vec{q}, \vec{p}, s m_{s}\left|t_{\pi N} G_{\pi N}\left[t_{\gamma \pi}\left(W_{\gamma N_{1}}\right)+t_{\gamma \pi}\left(W_{\gamma N_{2}}\right)\right]\right| 1 m_{d}\right\rangle,
\end{aligned}
$$

where $t_{N N}$ and $t_{\pi N}$ denote respectively the $N N$ and $\pi N$ scattering matrices and $G_{N N}$ and $G_{\pi N}$ the corresponding free two-body propagators. For the actual evaluation, the scattering matrices are expanded into partial waves, and the expansion is then truncated at a certain angular momentum such that convergence is achieved.

\section{B. The differential cross section including polarization asymmetries}

The general five-fold differential cross section $d^{5} \sigma / d q d \Omega_{q} d \Omega_{p}$ including beam and target polarization has been derived in [23], and we refer to this work for details. In the present work we are interested in the semi-exclusive reaction $\vec{d}(\vec{\gamma}, \pi) N N$ where only the produced pion is detected. This means integration of the five-fold differential cross section over $\Omega_{p}$, yielding as semi-exclusive differential cross section [23]

$$
\begin{aligned}
\frac{d^{3} \sigma}{d q d \Omega_{q}}= & \frac{d^{3} \sigma_{0}}{d q d \Omega_{q}}\left[1+P_{l}^{\gamma}\left\{\widetilde{\Sigma}^{l} \cos 2 \phi_{q}+\sum_{I=1}^{2} P_{I}^{d} \sum_{M=-I}^{I} \widetilde{T}_{I M}^{l} \cos \left[M \phi_{q d}-2 \phi_{q}-\delta_{I 1} \pi / 2\right] d_{M 0}^{I}\left(\theta_{d}\right)\right\}\right. \\
& \left.+\sum_{I=1}^{2} P_{I}^{d} \sum_{M=0}^{I}\left(\widetilde{T}_{I M}^{0} \cos \left[M \phi_{q d}-\delta_{I 1} \pi / 2\right]+P_{c}^{\gamma} \widetilde{T}_{I M}^{c} \sin \left[M \phi_{q d}+\delta_{I 1} \pi / 2\right]\right) d_{M 0}^{I}\left(\theta_{d}\right)\right]
\end{aligned}
$$

where $\phi_{q d}=\phi_{q}-\phi_{d}$. Explicit expressions for the asymmetries $\widetilde{\Sigma}^{l}, \widetilde{T}_{I M}$, and $\widetilde{T}_{I M}^{c / l}$ are listed in the appendix of [23]. Furthermore, the photon polarization is characterized by the degree of circular polarization $P_{c}^{\gamma}$ and the degree of linear polarization $P_{l}^{\gamma}$, where the $x$-axis has been chosen in the direction of maximum linear polarization. The deuteron target is characterized by four parameters, namely the vector and tensor polarization parameters $P_{1}^{d}$ and $P_{2}^{d}$, respectively, and by the orientation angles $\theta_{d}$ and $\phi_{d}$ of the deuteron orientation axis with respect to which the deuteron density matrix has been assumed to be diagonal. 
We would like to point out that in forward and backward pion emission the following asymmetries vanish at $\theta_{q}=0$ or $\pi$

$$
\widetilde{\Sigma}^{l}=0, \quad \widetilde{T}_{I M}^{0, c}=0 \text { for } M \neq 0, \quad \text { and } \quad T_{I M}^{l}=0 \text { for } M \neq 2,
$$

because of helicity conservation, i.e. in this case the cross section should not depend on $\phi_{q}$.

In the next section we will present results for the case that only the direction of the outgoing pion is measured and not its momentum. Then the corresponding differential cross section $d^{2} \sigma / d \Omega_{q}$ is given by an expression formally analogous to (9), where unpolarized cross section and asymmetries are replaced by

$$
\begin{aligned}
\frac{d^{3} \sigma_{0}}{d q d \Omega_{q}} & \rightarrow \frac{d^{2} \sigma_{0}}{d \Omega_{q}}=\int_{q_{\min }\left(\theta_{q}\right)}^{q_{\max }\left(\theta_{q}\right)} d q \frac{d^{3} \sigma_{0}}{d q d \Omega_{q}} \\
\frac{d^{3} \sigma_{0}}{d q d \Omega_{q}} \widetilde{\Sigma}^{l}\left(q, \theta_{q}\right) & \rightarrow \frac{d^{2} \sigma_{0}}{d \Omega_{q}} \Sigma^{l}\left(\theta_{q}\right)=\int_{q_{\min }\left(\theta_{q}\right)}^{q_{\max }\left(\theta_{q}\right)} d q \frac{d^{3} \sigma_{0}}{d q d \Omega_{q}} \widetilde{\Sigma}^{l}\left(q, \theta_{q}\right), \\
\frac{d^{3} \sigma_{0}}{d q d \Omega_{q}} \widetilde{T}_{I M}^{\alpha}\left(q, \theta_{q}\right) & \rightarrow \frac{d^{2} \sigma_{0}}{d \Omega_{q}} T_{I M}^{\alpha}\left(\theta_{q}\right)=\int_{q_{\min }\left(\theta_{q}\right)}^{q_{\max }\left(\theta_{q}\right)} d q \frac{d^{3} \sigma_{0}}{d q d \Omega_{q}} \widetilde{T}_{I M}^{\alpha}\left(q, \theta_{q}\right), \quad \alpha \in\{0, l, c\} .
\end{aligned}
$$

The upper and lower integration limits are given by

$$
\begin{aligned}
q_{\max }\left(\theta_{q}\right) & =\frac{1}{2 b}\left(a \omega \cos \theta_{q}+E_{\gamma d} \sqrt{a^{2}-4 b m_{\pi}^{2}}\right), \\
q_{\min }\left(\theta_{q}\right) & =\max \left\{0, \frac{1}{2 b}\left(a \omega \cos \theta_{q}-E_{\gamma d} \sqrt{a^{2}-4 b m_{\pi}^{2}}\right)\right\}
\end{aligned}
$$

where

$$
\begin{aligned}
a & =W_{\gamma d}^{2}+m_{\pi}^{2}-4 m_{N}^{2}, \\
b & =W_{\gamma d}^{2}+\omega^{2} \sin ^{2} \theta_{q}, \\
W_{\gamma d}^{2} & =m_{d}\left(m_{d}+2 \omega\right), \\
E_{\gamma d} & =m_{d}+\omega .
\end{aligned}
$$

Finally, in the total cross section only a few polarization observables survive, namely one has [23]

$$
\sigma\left(P_{l}^{\gamma}, P_{c}^{\gamma}, P_{1}^{d}, P_{2}^{d}\right)=\sigma_{0}\left[1+P_{2}^{d} \bar{T}_{20}^{0} \frac{1}{2}\left(3 \cos ^{2} \theta_{d}-1\right)+P_{c}^{\gamma} P_{1}^{d} \bar{T}_{10}^{c} \cos \theta_{d}+P_{l}^{\gamma} P_{2}^{d} \bar{T}_{22}^{l} \cos \left(2 \phi_{d}\right) \frac{\sqrt{6}}{4} \sin ^{2} \theta_{d}\right]
$$

where unpolarized total cross section and asymmetries are given by

$$
\begin{gathered}
\sigma_{0}=\int d \Omega_{q} \int_{q_{\min }\left(\theta_{q}\right)}^{q_{\max }\left(\theta_{q}\right)} d q \frac{d^{3} \sigma_{0}}{d q d \Omega_{q}} \\
\bar{\sigma}_{0} \bar{T}_{I M}^{\alpha}=\int d \Omega_{q} \int_{q_{\min }\left(\theta_{q}\right)}^{q_{\max }\left(\theta_{q}\right)} d q \frac{d^{3} \sigma_{0}}{d q d \Omega_{q}} \widetilde{T}_{I M}^{\alpha}
\end{gathered}
$$

with $\alpha \in\{0, l, c\}$. This concludes the formal part.

\section{RESULTS AND DISCUSSION}

For the calculation of the $N N$-rescattering contribution we have taken the separable representation of the realistic Paris potential from [26] and included all partial waves up to ${ }^{3} D_{3}$. Also the deuteron wave function was calculated using this potential. In principle, any other realistic potential, e.g. the Bonn r-space potential [11], could be used as well, because the results do not depend sensitively on the potential model as was found in [14]. However, one comment is in order with respect to the question, whether the use of such a nonrelativistic $N N$-potential can be justified in view of the high energies involved, because the potential is fit to $N N$-scattering data up to nucleon lab kinetic energies of $T_{N N}=330 \mathrm{MeV}$ only. But even up to $T_{N N}=500 \mathrm{MeV}$, it reproduces the phase shifts reasonably well because the inelasticity parameters are still small. Since the calculation of $N N$-rescattering requires $N N$-scattering amplitudes also at considerably higher energies, where we still use the same separable representation, one might expect some 
serious error. On the other hand, the size of this error depends on the relative size of that part of the phase space, where the energy of the $N N$-subsystem exceeds the region of validity. In order to estimate the corresponding error, we present in Fig. 2 the cross section $d \sigma / d T_{N}$ as a function of the equivalent nucleon kinetic lab energy $T_{N}$ of $N N$ scattering. One readily notes that even for a photon energy of $800 \mathrm{MeV}$ the dominant part of the cross section corresponds to nucleon laboratory kinetic energies of less than $500 \mathrm{MeV}$. Thus, the use of such a realistic potential is justified.

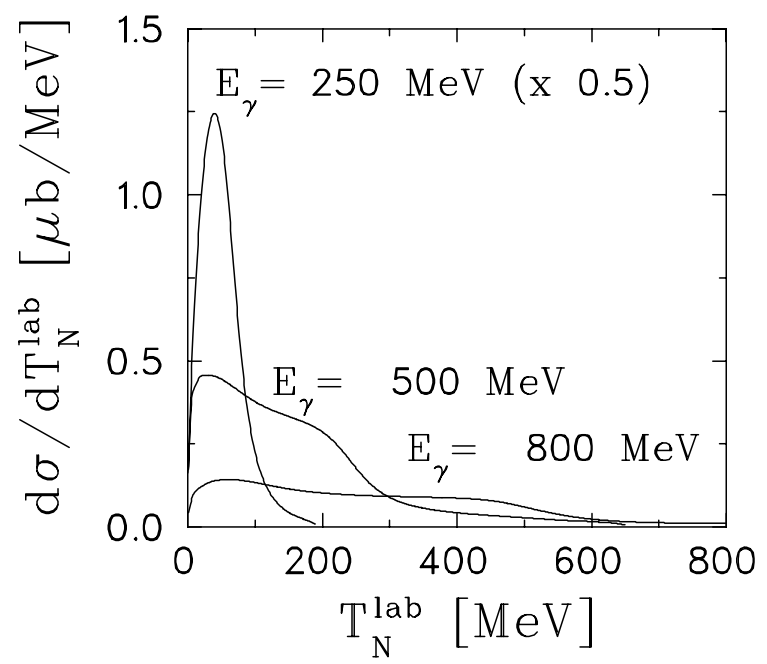

FIG. 2: Semi-exclusive differential cross section $d \sigma / d T_{N}$ in IA with respect to the equivalent nucleon laboratory kinetic energy $T_{N}$ of $N N$-scattering for three photon energies $E_{\gamma}=250,500$ and $800 \mathrm{MeV}$.

Similarly, $\pi N$-rescattering is evaluated using a realistic separable representation of the $\pi N$-interaction from 27] and taking into account all partial waves up to $l=2$. We have evaluated the semi-exclusive differential cross section including the various polarization asymmetries in IA alone and with inclusion of $N N$ - and $\pi N$-rescattering. As already mentioned, the elementary pion photoproduction amplitude is taken from the MAID model. Since it is parametrized in terms of the Chew-Goldberger-Low-Nambu amplitudes (CGLN) [28], defined in the $\gamma N$ c.m. frame, we first had to transform this amplitude into a general frame of reference. This is achieved by introducing invariant amplitudes and establishing relations to the CGLN amplitudes in the c.m. frame. This is described in detail in [29] and needs not to be repeated here. Furthermore, for the evaluation of the MAID amplitudes the invariant $\pi N$-energy and the pion angle in the $\pi N$ c.m. system have to be specified. For this purpose we assume that the four-momenta $q$ and $p_{f}$ of pion and active nucleon in the final state obey the on-shell condition. Then the corresponding c.m. variables are obtained by a Lorentz transformation with $\vec{\beta}=-\left(\vec{q}+\vec{p}_{f}\right) /\left(w_{\pi}+E_{f}\right)$. The initial four-momentum $p_{i}$ of the active nucleon is determined by assuming four-momentum conservation at the elementary vertex, i.e. $p_{i}=q+p_{f}-k$.

\section{A. Total cross sections}

We begin the discussion of the results with the total cross sections for the three charge states displayed in Fig. 3 . where we have plotted in addition the corresponding elementary cross section for comparison. The threshold region

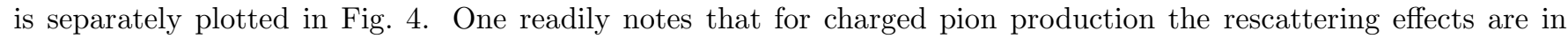
general quite small. Only close to threshold they lead to a sizeable enhancement (see left panel of Fig. (4), mainly by $N N$-rescattering while $\pi N$-rescattering is almost negligible.

The significant role of $N N$-FSI in photoproduction of mesons at very low energies has been noted previously in [5, 25, 30]. We would only like to mention that this effect has a kinematical rather than a dynamical origin. As pointed out in 25], in IA the energy needed for pion production below the free nucleon threshold is provided exclusively through the high momentum of a nucleon moving towards the incoming photon. As a result, the IA predicts an anomalous suppression of the cross section, because of a small probability for finding a nucleon with high momentum in the deuteron wave function. Thus, $N N$-rescattering provides a mechanism to balance the strong mismatch between the momentum needed to produce the pion and the characteristic internal nucleon momentum in the deuteron, so that the strong suppression appearing in IA can be avoided. The same reasoning is true for $\pi^{0}$ channel. But in this case the below mentioned role of orthogonality turns out to be very important so that the resulting FSI effect becomes destructive already at $10 \mathrm{MeV}$ and higher above threshold. 

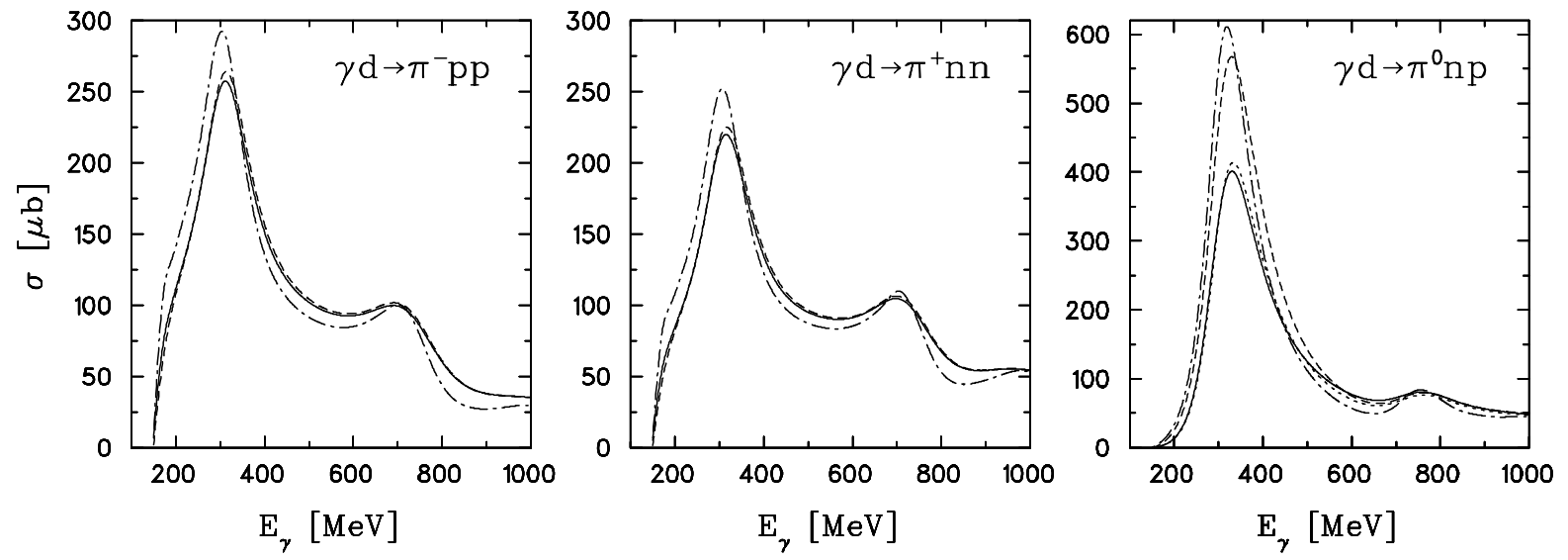

FIG. 3: Total cross section for pion photoproduction on the deuteron. Notation of curves: dashed: IA; solid: IA $+N N-$ and $\pi N$-rescattering; dash-dot: corresponding elementary cross section. For $\pi^{0}$-production: dotted curve describes the modified IA.
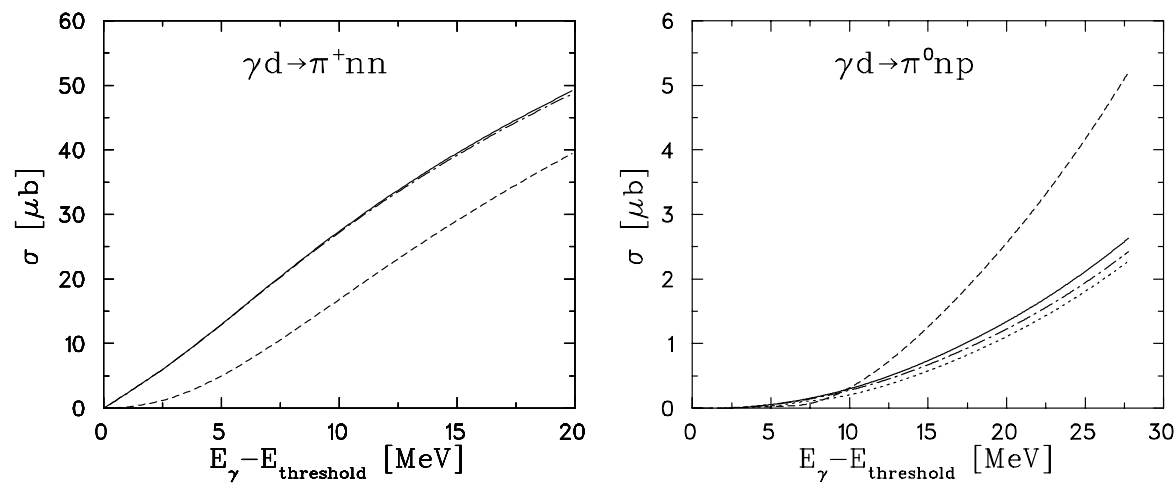

FIG. 4: Total cross section near threshold for $\pi^{+}$- and $\pi^{0}$-photoproduction on the deuteron. Notation of curves: dashed: IA; dash-dot: IA $+N N$-rescattering; solid: IA $+N N$ - and $\pi N$-rescattering; dotted in the right panel: modified IA.

At higher energies, near and above the maximum the cross sections of charged pion production are reduced by FSI by a few percent. Therefore, the main difference to the elementary cross section comes from the Fermi motion leading to a slight reduction and a shift of the maximum, and a broadening of the whole distribution. As one can see in Fig. [3] the result of these features is that the energy integrated cross section

$$
I(E)=\int_{E_{t h}}^{E} \sigma\left(E_{\gamma}\right) d E_{\gamma}
$$

as function of the upper integration limit $E$, is preserved over a wide energy region. In other words, the integral at $E=1 \mathrm{GeV}$ has approximately the same value for the reaction on the free nucleon and on the deuteron. Concerning the role of FSI in the $\pi^{ \pm}$channels at higher energies, one can assume that the interaction between the emitted particles leads basically to a redistribution of events in phase space, so that in the absence of absorption the overall yield of particles does not change and the FSI effect in the total cross section remains insignificant. It is, however, not the case for the near threshold region where, as was discussed above, the IA amplitude turns out to be anomalously suppressed in the available phase space.

In contrast to charged pion production, one notes quite large FSI effects from $N N$-rescattering in $\pi^{0}$-production as displayed in the right panel of Fig. 3 However, these effects are mainly due to the elimination of the spurious coherent contribution in the IA. That is nicely demonstrated by the results for the modified IA, also shown in Fig. 3] where the deuteron wave function component in the final plane wave is projected out (see Appendix $\mathbb{B}$ for details). The additional rescattering contributions beyond the orthogonality effect are comparable to what was found in charged pion production, except near threshold where one finds a much smaller influence of FSI. With respect to the elementary cross section, one notes a sizeable reduction for incoherent $\pi^{0}$-production on the deuteron, because part of the strength goes to the coherent channel $d\left(\gamma, \pi^{0}\right) d$. The spurious admixture of the coherent channel in the IA cross section was 

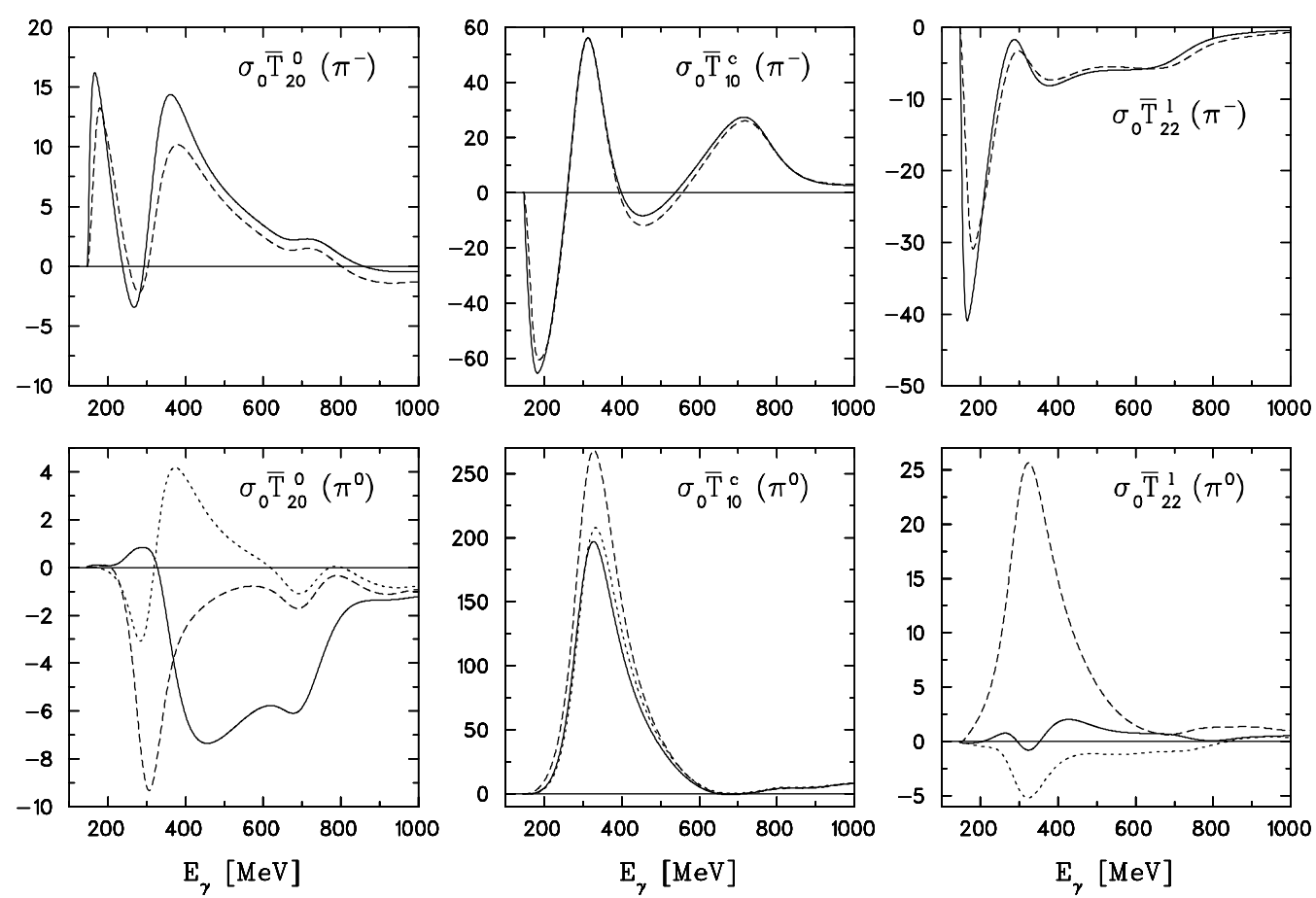

FIG. 5: Polarization asymmetries $\sigma_{0} \bar{T}_{20}^{0}, \sigma_{0} \bar{T}_{10}^{c}$, and $\sigma_{0} \bar{T}_{22}^{l}$ of the total cross section for $\pi^{-}$- (upper panels) and $\pi^{0}$ photoproduction (lower panels) on the deuteron. Notation of curves: dashed: IA; solid: IA $+N N$-rescattering; For $\pi^{0}$ production: dotted curve describes the modified IA.

also discussed in 31].

The polarization observables of the total cross section are shown in Fig. 5 only for $\pi^{-}$- and $\pi^{0}$-production since the ones for $\pi^{+}$- and $\pi^{-}$-production are quite similar. The vector asymmetry for circularly polarized photons $\sigma_{0} \bar{T}_{10}^{c}$ (middle panels), which governs the spin asymmetry of the GDH sum rule, reproduces the results reported in [24]. The sensitivity to FSI is weak, similar to the total cross sections. The tensor asymmetries for $\pi^{-}$-production are sizeable in the near threshold region, exhibiting a sharp peak in absolute values. $\sigma_{0} \bar{T}_{20}^{0}$ reaches a second quite broad maximum above the $\Delta$-resonance, while $\sigma_{0} \bar{T}_{22}^{l}$ remains small. FSI is more notable than in the total cross section. For $\pi^{0}$ production, the tensor asymmetries show quite a different behavior compared to $\pi^{-}$-production. They are quite small in general, almost vanishing in the near threshold region. Furthermore, they show huge FSI effects beyond the orthogonality effect.

\section{B. Differential cross section}

Angular distributions are shown in Fig. [6 for $\pi^{-}$-production and in Fig. 7 for $\pi^{0}$-production. Like for the total cross section, one notes for $\pi^{-}$-production a small size of rescattering effects. At the lowest energy $(250 \mathrm{MeV})$ they decrease the cross section at forward angles and increase it in backward direction. At $350 \mathrm{MeV}$ one finds a slight enhancement near the maximum, while at the two higher energies a sizeable decrease is found only in the extreme forward direction. For comparison, we show also the elementary cross sections, and again the smoothing effect of the Fermi motion is apparent.

Compared our calculation with the predictions of [5] and [8] we note a significant disagreement for the IA at forward angles. At $E_{\gamma}=500 \mathrm{MeV}$ our IA cross section exhibits a visible rise which is governed mainly by the pion photoelectric term in the elementary amplitude. At the same time the calculations of [8] (see dotted curve in the lower left panel of Fig. [ at $E_{\gamma}=500 \mathrm{MeV}$ ) predict a strong reduction in the same angular region, so that the corresponding IA result at $\theta_{\pi}=0$ is about 10 times smaller than ours.

A possible reason of this discrepancy has been discussed in [14]. Namely, it was claimed in [8] that the drastic reduction of the cross section compared to the elementary reaction is a manifestation of the Pauli principle, leading to a strong suppression of the $p p$ states in the region of small relative momenta in this subsystem. On the contrary, we would like to note that this suppression takes place only in the triplet state $(s=1)$ of the emitted protons, whereas 

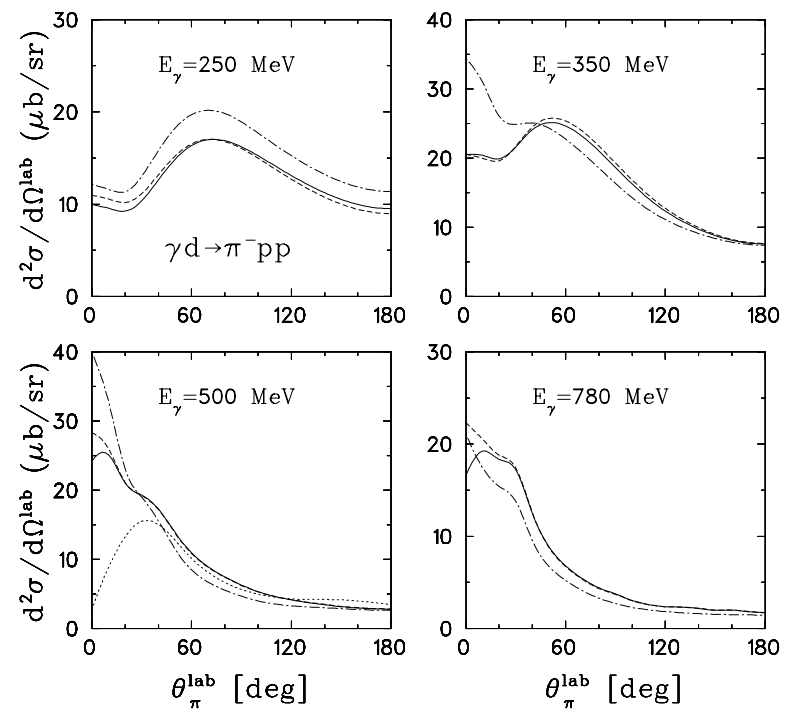

FIG. 6: Semi-exclusive differential cross section for $\pi^{-}$photoproduction on the deuteron. Notation of curves: dashed: IA; solid: IA $+N N$-rescattering; dash-dot: elementary process $n\left(\gamma, \pi^{-}\right) p$; dotted in lower left panel: IA from [8].

the singlet part $(s=0)$, where the processes on the individual nucleons can coherently enhance each other, peaks at $\theta_{\pi}=0$ similar to the elementary cross section. The resulting angular distribution shows some reduction at forward angles, compared to the elementary reaction, which, however, is not as large as that exhibited by the triplet part only and is, therefore, much smaller compared to the prediction of [5, 8].

On the other hand, inclusion of FSI leads in [5, 8] to a significant increase at small angles compared to the IA cross section, whereas in our case the effect of FSI is small and destructive. It is, therefore, interesting to note that the difference between the full results of the present work (see Fig. 24 below) and of [8] is rather small, so that both models describe the data equally well.
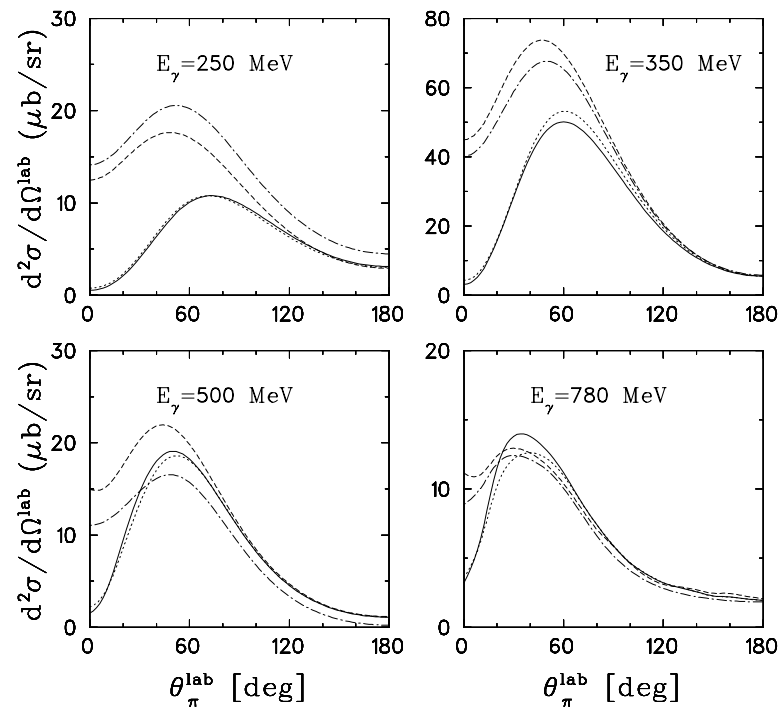

FIG. 7: Semi-exclusive differential cross section for $\pi^{0}$ photoproduction on the deuteron. Notation of curves: dashed: IA; dotted: modified IA; solid: IA $+N N$-rescattering; dash-dot: average elementary process on neutron and proton.

As was mentioned above, in the $\pi^{0}$ channel the orthogonality effect appears so that the role of FSI is approximately determined by the fraction of the coherent reaction $\gamma d \rightarrow \pi^{0} d$ in the inclusive $\pi^{0}$ photoproduction. Fig. [7 demonstrates huge spurious contribution of the coherent process in IA which is eliminated by applying the modified IA. This result could be expected because the effect of orthogonality should be especially visible in the region of small pion angles, where the momentum transferred to the nucleons is minimal and the overlap between initial and final wave functions is most important. The additional FSI effects are quite small. Only at $350 \mathrm{MeV}$ one notes a slight increase of the 
cross section near the maximum around $60^{\circ}$ and at $780 \mathrm{MeV}$ a more sizeable increase.

\section{Beam asymmetry for linearly polarized photons}

As next we turn to the beam asymmetry $\Sigma^{l}$ displayed in Fig. 8 for the three charge states at various energies in the $\Delta$-resonance region and above. In all of these asymmetries one notes a relatively small influence from FSI. We will consider first the results for $\pi^{-}$-production in the top panels of Fig. 8 For the lowest three energies the photon asymmetry $\Sigma^{l}$ is negative. It is quite small below the $\Delta$-region and increases considerably in absolute size with increasing energy, becoming quite broad around $350 \mathrm{Mev}$ but more forward peaked at $500 \mathrm{Mev}$. At the highest energy of $780 \mathrm{MeV}$ one notes a different behavior. The deep minimum in forward direction turns into a broad positive distribution above $40^{\circ}$. The three lowest energies are also representative for $\pi^{+}$-production (middle panels of Fig. [8). However at the highest energy the asymmetry remains negative, but exhibits a secondary minimum around $90^{\circ}$.
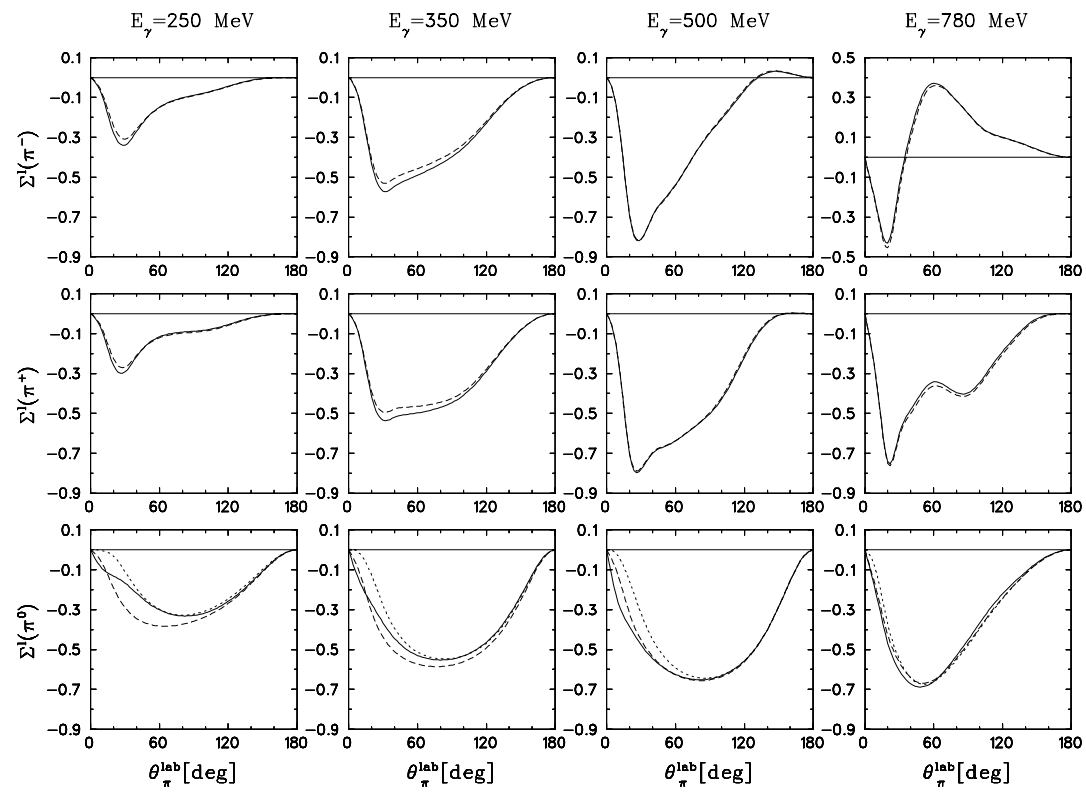

FIG. 8: Photon asymmetry $\Sigma^{l}$ for the three charge states of semi-exclusive single pion photoproduction on the deuteron. Notation of curves: dashed: IA; dotted: modified IA for $\pi^{0}$-production; solid: IA $+N N$-rescattering.

The $\Sigma^{l}$-asymmetry for $\pi^{0}$-production, shown in the lowest panels of Fig. [8 exhibits quite a different behavior compared to charged pion production. One finds a broad, structureless and sinus-shaped negative distribution with a maximum around $80^{\circ}$ to $90^{\circ}$ with increasing amplitude by about a factor of two going from 250 to $500 \mathrm{MeV}$. At $780 \mathrm{MeV}$ the width of the distribution becomes smaller and the minimum moves towards forward angles. In general also here the influence of rescattering is small. It is mainly due to the removal of the spurious coherent contribution. The relatively largest influence appears at the lowest energy of $E_{\gamma}=250 \mathrm{MeV}$.

Compared to the results in 18, 19, 21 one readily notes quite substantial differences for both, charged and neutral pion production, both in angular behavior and also in absolute size, in particular at higher energies. For $\pi^{-}$-production a larger influence of FSI is found in [22] which we cannot confirm. Furthermore, $\Sigma^{l}$ does not vanish in [22] at $\theta_{q}=0$ and $\pi$ as it should, although it is small. Strangely enough, the authors mention this feature as a notable effect. The origin of these differences is not clear. In any case, the comparison suffers from the questionable formal expressions used for the calculation of $\Sigma^{l}$ which, moreover, differ in the various publications [18, 21, 22].

\section{Target asymmetries for oriented deuteron}

The target asymmetries $T_{I M}^{0}$ are shown in Figs. 9 through 11 for the three charge states, respectively. In view of the similarity of the asymmetries for the two charge states except for $T_{11}^{0}$ at $780 \mathrm{MeV}$, we will restrict the discussion to $\pi^{-}$- and $\pi^{0}$-production. In general FSI effects are again small, the largest appear in $T_{21}^{0}$. The vector asymmetry $T_{11}^{0}$ is positive up to $500 \mathrm{MeV}$ and shows a broad distribution over the whole angular range. But at $780 \mathrm{MeV}$ an oscillatory behavior develops with sizeable amplitude. Only at the lowest energy of $E_{\gamma}=250 \mathrm{MeV}$ one notes some 

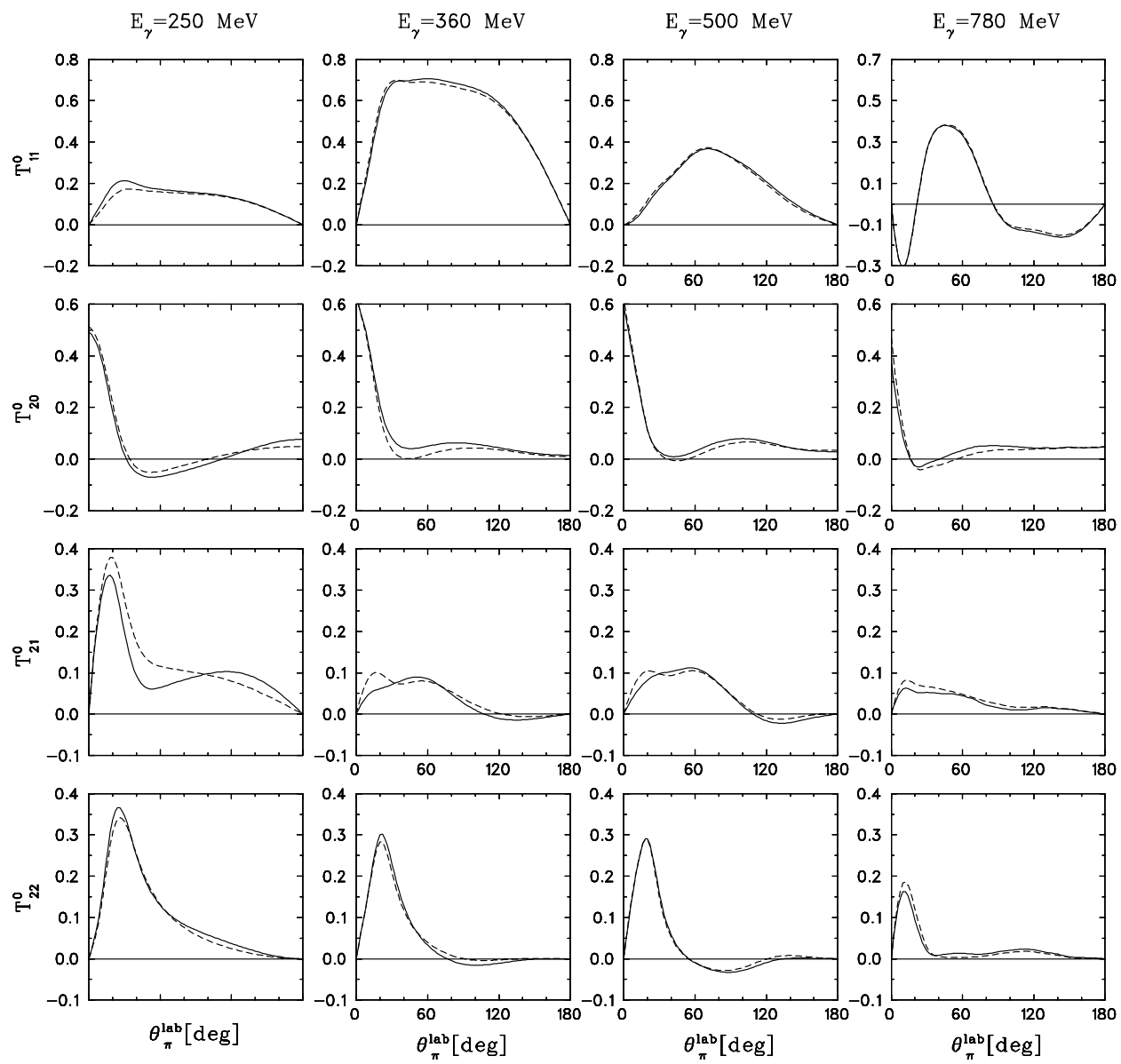

FIG. 9: Target asymmetries $T_{I M}^{0}$ for semi-exclusive $\pi^{-}$-photoproduction on the deuteron. Notation of curves as in Fig. 8

notable influence at forward angles from FSI. With respect to [18, 19, 22] we find an opposite sign, a smaller amplitude and also a somewhat different angular behavior.

The tensor target asymmetry $T_{20}^{0}$ exhibits a pronounced sharp peak at $0^{\circ}$ and a rapid fall-off with increasing angles, remaining quite small above $30^{\circ}$. The results are similar to $18,19,22$ except that in contrast to the small negative values of [18, 19] at backward angles, we find small positive values. Moreover, we find smaller FSI influences at backward angles than in [22]. $T_{21}^{0}$ peaks at small angles around $20^{\circ}$ for $E_{\gamma}=250 \mathrm{MeV}$ which disappears at the three higher energies becoming a broader distribution with a considerably smaller size. FSI shows some notable influence. Again we find significant differences to [18, 19] with respect to shape, size, and FSI effects, in particular at the lowest energy. Finally, $T_{22}^{0}$ exhibits a prominent peak in forward direction which becomes sharper and moves towards smaller angles with increasing photon energy. This is in qualitative agreement with [18, 19] although the size is different and our FSI effects are much smaller.

The target asymmetries for $\pi^{0}$-production in Fig. 11] show quite a different behavior compared to charged pion production. The structure of $T_{11}^{0}$ changes significantly with energy. While at $E_{\gamma}=250 \mathrm{MeV}$ one finds a maximum around $130^{\circ}$, one notes a forward negative minimum and a backward positive maximum at $E_{\gamma}=350 \mathrm{MeV}$, at $E_{\gamma}=500 \mathrm{MeV}$ a broad and quite flat negative distribution, and finally at $780 \mathrm{MeV}$ a forward maximum and a negative minimum around $80^{\circ}$. The tensor asymmetries are much more sensitive to FSI. This is particularly apparent in $T_{20}^{0}$ exhibiting a forward negative minimum in IA which turns into a positive forward peak when FSI is switched on. Also $T_{21}^{0}$ shows such a drastic influence from FSI. $T_{22}^{0}$ is much smaller than for charged pion production and shows an oscillatory behavior. FSI effects are noticeable again. For these asymmetries the differences to the results of [18, 19] are again quite significant. 

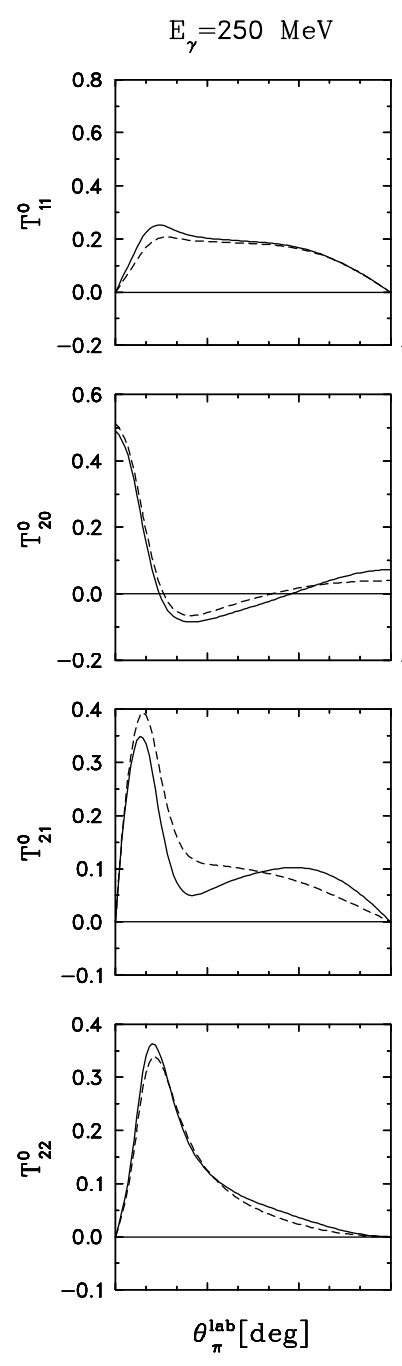

$\mathrm{E}_{\gamma}=360 \mathrm{MeV}$
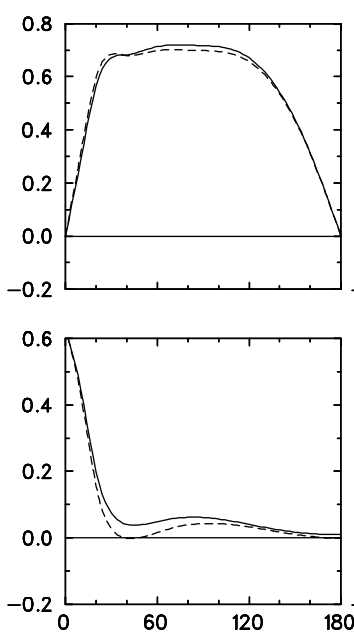

$\mathrm{E}_{\gamma}=500 \mathrm{MeV}$
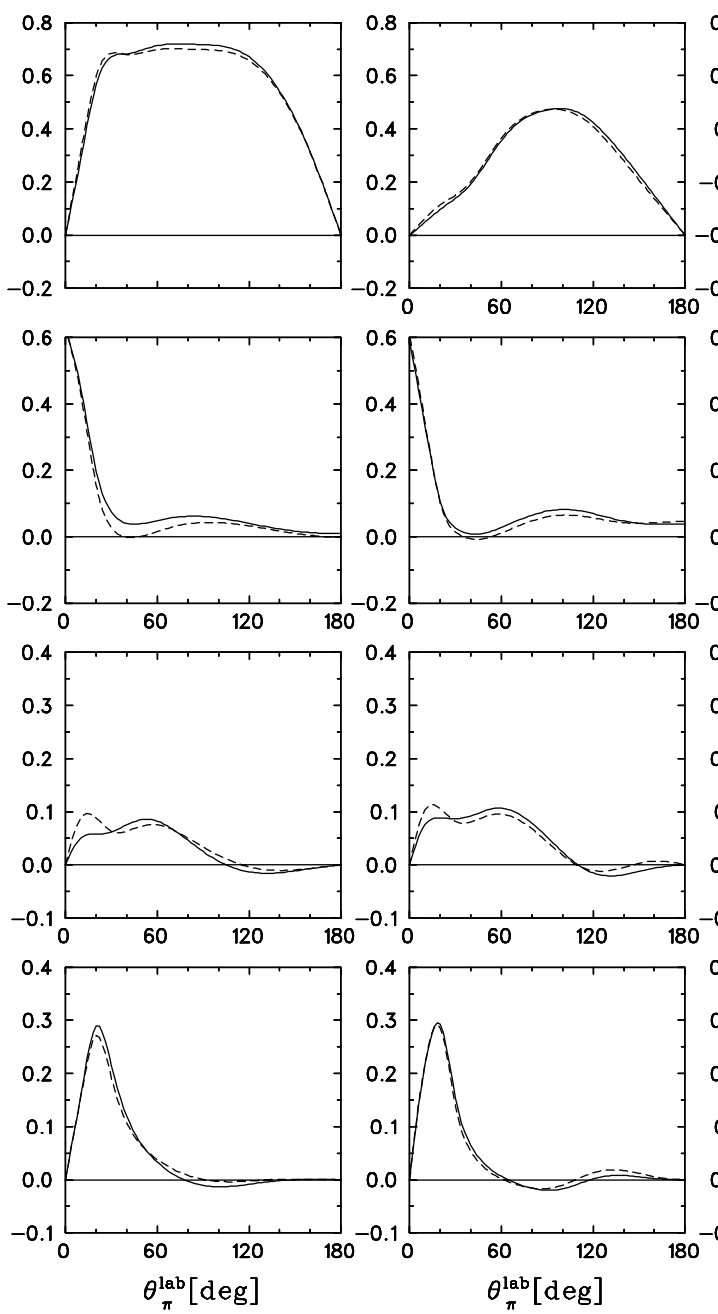
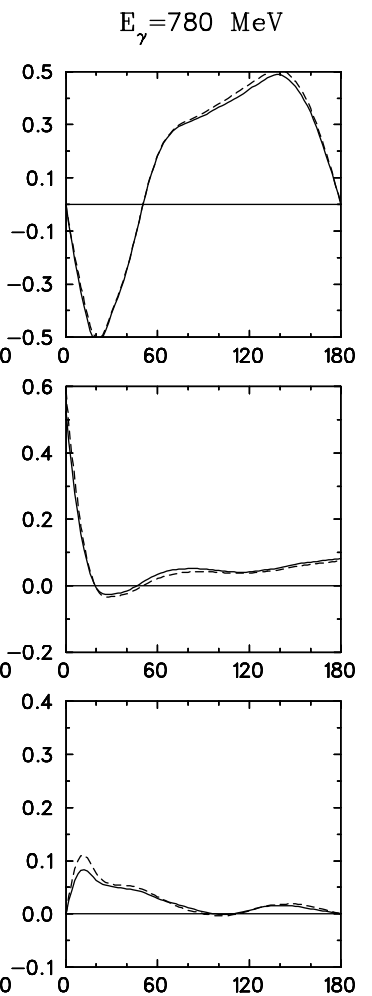

FIG. 10: Target asymmetries $T_{I M}^{0}$ for semi-exclusive $\pi^{+}$-photoproduction on the deuteron. Notation of curves as in Fig. 8

\section{E. Beam-target asymmetries for circularly polarized photons}

As next we will discuss the double polarization asymmetries $T_{I M}^{c}$ for circularly polarized photons and oriented deuterons. They are in a certain sense complementary to the target asymmetries $T_{I M}^{0}$ because, while one is the real part, the other is the imaginary part of the basic quantities $V_{I M}^{1}$ as defined in [23]. Since the results for the two charged pions are again in general quite similar, we display the results only for $\pi^{-}$in Fig. 12 and for $\pi^{0}$ in Fig. 13, In contrast to what has been claimed in [19], all of them are nonvanishing. As a sideremark, although $T_{10}^{c}$ should vanish according to [19], a non-vanishing spin asymmetry is reported in [20]. For both, $\pi^{0}$ and $\pi^{-}$, the vector asymmetry $T_{10}^{c}$ is quite sizeable in forward and backward direction and also around $90^{\circ}$ for $\pi^{0}$. It is this asymmetry which determines the Gerasimov-Drell-Hearn sum rule. The influence of FSI is quite marginal, in particular for $\pi^{-}$. For both charge states the energy dependence is weak. The other vector asymmetry $T_{11}^{c}$ shows a rather different behavior. For $\pi^{-}$it is predominantly negative with a slight preference of the backward direction, especially at higher energies, while for $\pi^{0}$ it has a positive maximum around $30^{\circ}$ and a negative minimum around $150^{\circ}$, almost independent of the energy. Considerably smaller are the tensor asymmetries $T_{21}^{c}$ and $T_{22}^{c}$, but they are a little more sensitive to FSI. This is particularly apparent for $T_{21}^{c}$ in $\pi^{0}$ production above the $\Delta$-resonance region. 

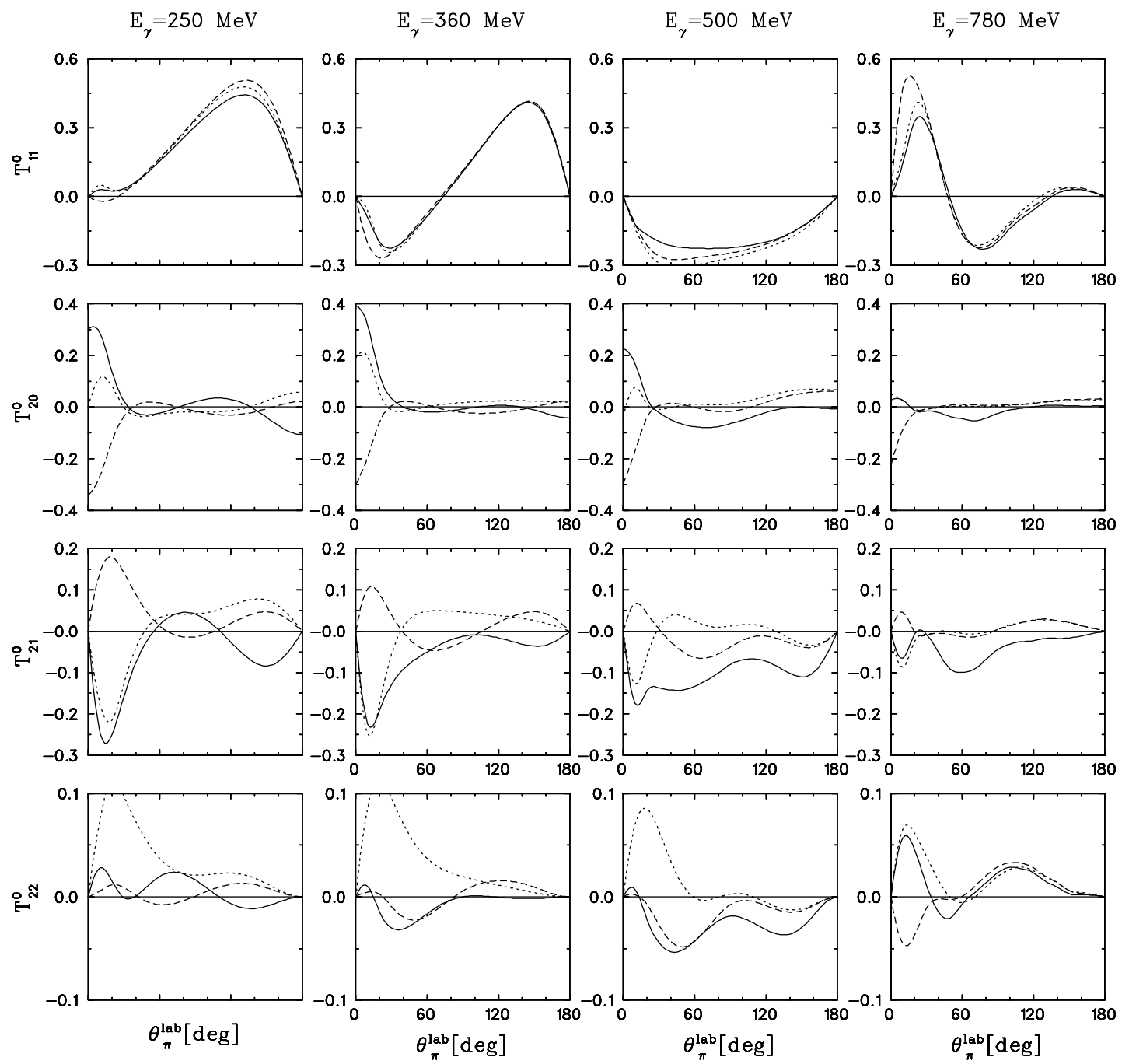

FIG. 11: Target asymmetries $T_{I M}^{0}$ for semi-exclusive $\pi^{0}$-photoproduction on the deuteron. Notation of curves as in Fig. 8

\section{F. Beam-target asymmetries for linearly polarized photons}

The beam-target asymmetries $T_{I M}^{l}$ for linearly polarized photons and polarized deuterons for $\pi^{-}$and $\pi^{0}$ production are shown in Figs. 14 through 17] Also these asymmetries are very similar for the two charge states $\pi^{-}$and $\pi^{+}$. The vector asymmetries $T_{1 M}^{l}$ do not vanish at all as claimed in [18]. They are small for $\pi^{-}$at the lowest energy of $E_{\gamma}=250 \mathrm{MeV}$, but become sizeable for the higher energies (see Fig. 144). They are considerably smaller for $\pi^{0}$ production as shown in Fig. [15] but more sensitive to FSI. Of the corresponding tensor asymmetries in Figs. [16] and [17 $T_{22}^{l}$ is by far the largest for both charge states $\pi^{-}$and $\pi^{0}$, exhibiting a pronounced forward peak which becomes slightly sharper with increasing energy. The size decreases significantly when going from $T_{22}^{l}$ to $T_{2-2}^{l}$. By the way, they are certainly not equal as claimed in [18]. While $T_{2-2}^{l}$ is restricted to vanish at $\theta_{q}=0$ and $\pi$, this is not the case for $T_{22}^{l}$. For $\pi^{-}$production one notes some FSI effects in $T_{2 \pm 1}^{l}$, whereas for $\pi^{0}$ quite drastic influences from FSI can be seen, leading even to a sign change in some of them. A comparison to the results in [18] makes no sense because of the above mentioned wrong formal expressions in [18].

\section{G. Comparison with experiment}

We now will turn to a comparison with experimental data, where available. Fig. 18] shows the total cross sections for $\pi^{-}$- and $\pi^{0}$-production. The agreement with experimental data for $\pi^{-}$-production is satisfactory although not perfect. The theory is a little high in the maximum but low in the dip region between the $\Delta$ and the second resonance 
region. For $\pi^{0}$, one notes some slight overshooting in the maximum and a sizeable overestimation above the $\Delta$ in the second resonance region. This discrepancy was already discussed in [33] where it was noted that the smearing and damping of the second resonance peak can hardly be explained by the Fermi motion effect alone. According to our results, the broadening of the resonance structure in pion production on the deuteron is quite significant. This effect is readily seen in Fig. 19, where we present differential cross sections for $\pi^{0}$-production on the deuteron as functions of the photon energy for fixed pion angle. The cross section refers to the equivalent $\gamma N$ c.m. system, where the nucleon is at rest in the deuteron. The solid curves are our full calculation multiplied by energy independent factors, whose values are listed in the various panels. One readily sees that the Fermi motion and to some extent FSI leads to a disappearing of the resonance structure. The ratio of the theoretical cross section to the experimental result is about 1.75, except for $\cos \theta_{\pi^{0}}^{*}=-0.9$ where it is about 3 . However the origin of this factor is unclear.

One possible source could be the neglected interaction of nucleon resonances with the spectator nucleon which might result in a broadening of the resonances due to additional inelastic processes. In fact, within a simple model calculation for the $\Delta$-resonance a significant lowering of the total cross section from such an interaction was found in [34]. A further source could be the neglected inelasticity of the final $N N$-interaction at higher energies. On the other hand the question arises, why the same lowering is not observed in the $\pi^{-}$-photoproduction where the agreement with the data is quite satisfactory.

The threshold region is shown in Fig. 20 for $\pi^{+}$-production. Good agreement with the data is achieved, comparable in quality to a recent precision calculation in chiral perturbation theory 36 . The crucial role of $N N$-FSI at low energies, leading to a strong enhancement over the IA, is furthermore demonstrated by the differential cross section with respect to the relative energy of the two final neutrons in $\pi^{+}$-production in Fig. 21 for two different kinematical situations, pion emission in more forward (left panel) and in more backward direction (right panel). The ${ }^{1} S_{0}$-state of $n n$-scattering near threshold, a manifestation of the so-called antibound state as companion to the deuteron, is nicely resolved and also quite well reproduced by the theory if $N N$-rescattering is included. A similar result has been reported in [38].

With respect to differential cross sections, a comparison for $\pi^{-}$-production is exhibited in Fig. 22 and for $\pi^{0}$ production in Fig. 23] For $\pi^{-}$-production one notes a satisfactory agreement with experimental data from Benz et al. [6], whereas for $\pi^{0}$-production one finds for the three highest energies a slight overestimation of the theory in the maximum and at forward angles an underestimation.

A comparison to recent data from the LEGS-collaboration (LEGS-exp.L3b) [39] on the unpolarized semi-exclusive differential cross section and $\Sigma^{l}$ for $\pi^{-}$production is shown in Fig. 24 for two energies near the $\Delta$-region. One notes very little influence from FSI and quite a good agreement of the theoretical description with the data. Our results on $\Sigma^{l}$ are similar to the IA calculation of Lee and Sato [40], but at variance to [21] where also larger FSI effect were quoted. But the latter results are questionable for reasons mentioned above. Another comparison for $\Sigma^{l}$ with data from the LEGS-collaboration [39] are exhibited in Fig. 25] for constant pion emission angle as function of the photon energy. In this case the size is underestimated by the theory, although FSI shifts the results for IA in the right direction but not enough. A much better almost perfect agreement with respect to $\Sigma^{l}$ is shown in Fig. 26 for $\pi^{0}$-production.

Finally, we show in Fig. 27 for $\pi^{-}$-production a comparison between theory and experiment for the semi-exclusive differential spin asymmetry $d^{2}\left(\sigma^{P}-\sigma^{A}\right) / d \Omega_{q}$ with respect to circularly polarized photons and the deuteron spin oriented parallel $(\mathrm{P})$ or antiparallel $(\mathrm{A})$ to the photon spin. This spin asymmetry is related to the beam-target asymmetry $T_{10}^{c}$ according to

$$
\frac{d^{2}\left(\sigma^{P}-\sigma^{A}\right)}{d \Omega_{q}}=\sqrt{6} \frac{d^{2} \sigma_{0}}{d \Omega_{q}} T_{10}^{c} .
$$

Compared to the predictions in [20] we find the depth of the minimum at $0^{\circ}$ almost independent of the energy and a small positive asymmetry between $30^{\circ}$ and $60^{\circ}$. This spin asymmetry has been measured by the A2-collaboration [41]. However, the analysis of the data is not yet completed. Preliminary data were shown in [20] without authorization and thus are not shown here. Compared to these, the agreement is quite satisfactory, although in the angular region, where the data are available, the spin asymmetry is very small, almost compatible with zero. Thus it would be very desirable to have additional data at more forward angles, where the theoretical asymmetry exhibits a pronounced minimum. This concludes the discussion of results.

\section{CONCLUSION AND OUTLOOK}

In the present work we have exploited the role of polarization observables in incoherent pion photoproduction on the deuteron with particular emphasis on the influence of final state interaction in the $N N$ - and $\pi N$-subsystems of 
the final state. In the unpolarized total and semi-exclusive differential cross section $d^{2} \sigma / d \Omega_{q}$, where only the direction of the produced pion is measured, the influence of final state rescattering is quite small for charged pion production for photon energies up to $1 \mathrm{GeV}$. For $\pi^{0}$-production the influence is much larger. However, the dominant part of FSIeffect arises from the removal of a spurious coherent contribution in the impulse approximation when $N N$-rescattering is switched on. This is demonstrated by a modified IA, where the deuteron wave function component in the final $N N$-plane wave is projected out. The remaining FSI-effect is comparable to charged pion production.

As polarization observables we have considered all beam, target and beam-target asymmetries of the semi-exclusive differential cross section. Many of them are quite sizeable, in particular the photon asymmetry $\Sigma^{l}$ and the various vector asymmetries. The tensor asymmetries are in general considerably smaller. They are often quite insensitive to final state rescattering. Only a few, $T_{21}$, and $T_{21}^{c}$, show a larger influence in charged pion production.

A very interesting and still open question concerns the disagreement between theoretical and experimental results for $\gamma d \rightarrow \pi^{0} n p$ in the second resonance region. Although our calculation explains the strong smearing of the resonance structure, the data are overestimated by about a factor of 1.5 (see Figs. 18 and 19). Hopefully, new measurements of the ratio

$$
R=\frac{d \sigma\left(\gamma, \pi^{0} n\right)}{d \sigma\left(\gamma, \pi^{0} p\right)}
$$

for quasifree photoproduction on neutrons and protons can clarify the situation. The old data from [42, 43], pointing to $R=1$ in the second resonance region, seem to be in disagreement with the results of [33].

Future theoretical improvements should be devoted to the inclusion of two-body effects in the photoproduction amplitude, e.g. the interaction between a resonance and the spectator nucleon, inclusion of inelasticities in the $N N$ interaction and the role of relativistic effects at higher energies. The problem of off-shell effects for the elementary amplitude is another unsolved task. Furthermore, there is urgent need for a unified description of single and double pion production.

\section{Acknowledgments}

We would like to thank Michael Schwamb for interesting discussions and a careful reading of the manuscript. This work was supported by the Deutsche Forschungsgemeinschaft (SFB 443).

\section{APPENDIX A: SEPARATION OF POLARIZATION ASYMMETRIES}

In this appendix we will discuss how the various polarization asymmetries of the semi-incusive differential cross section can be separated by a proper variation of the photon polarization parameters $\left(P_{l}^{\gamma}\right.$ and $\left.P_{c}^{\gamma}\right)$, the deuteron polarization parameters $\left(P_{1}^{d}\right.$ and $\left.P_{2}^{d}\right)$, the polarization angles $\left(\theta_{d}\right.$ and $\left.\phi_{d}\right)$, and the dependence on the pion azimuthal angle $\phi_{q}$, similar to what has been described in [44]. To this end we write the differential cross section of (9) as follows

$$
S\left(P_{l}^{\gamma}, P_{c}^{\gamma}, P_{1}^{d}, P_{2}^{d}\right)=S_{0}\left[1+P_{1}^{d} A_{d}^{V}+P_{2}^{d} A_{d}^{T}+P_{l}^{\gamma}\left(A_{\gamma}^{l}+P_{1}^{d} A_{\gamma d}^{l V}+P_{2}^{d} A_{\gamma d}^{l T}\right)+P_{c}^{\gamma}\left(P_{1}^{d} A_{\gamma d}^{c V}+P_{2}^{d} A_{\gamma d}^{c T}\right)\right]
$$

with $S_{0}=S(0,0,0,0)$ as unpolarized differential cross section. Furthermore, we have introduced as generalized single polarization $A$-asymmetries

$$
\begin{aligned}
A_{d}^{V}\left(\theta_{q}, \phi_{q d}, \theta_{d}\right) & =\widetilde{T}_{11}^{0}\left(\theta_{q}\right) \sin \phi_{q d} d_{10}^{1}\left(\theta_{d}\right) \\
A_{d}^{T}\left(\theta_{q}, \phi_{q}, \phi_{q d}, \theta_{d}\right) & =\sum_{M=0}^{2} \widetilde{T}_{2 M}^{0}\left(\theta_{q}\right) \cos \left(M \phi_{q d}\right) d_{M 0}^{2}\left(\theta_{d}\right), \\
A_{\gamma}^{l}\left(\theta_{q}, \phi_{q}\right) & =\widetilde{\Sigma}^{l}\left(\theta_{q}\right) \cos 2 \phi_{q}
\end{aligned}
$$


and double polarization $A$-asymmetries

$$
\begin{aligned}
A_{\gamma d}^{l V}\left(\theta_{q}, \phi_{q}, \phi_{q d}, \theta_{d}\right) & =\sum_{M=-1}^{1} \widetilde{T}_{1 M}^{l}\left(\theta_{q}\right) \sin \left(M \phi_{q d}-2 \phi_{q}\right) d_{M 0}^{1}\left(\theta_{d}\right) \\
& =\sum_{M=-1}^{1} \widetilde{T}_{1 M}^{l}\left(\theta_{q}\right)\left[\sin \left(M \phi_{q d}\right) \cos \left(2 \phi_{q}\right)-\cos \left(M \phi_{q d}\right) \sin \left(2 \phi_{q}\right)\right] d_{M 0}^{1}\left(\theta_{d}\right), \\
A_{\gamma d}^{l T}\left(\theta_{q}, \phi_{q}, \phi_{q d}, \theta_{d}\right) & =\sum_{M=-2}^{2} \widetilde{T}_{2 M}^{l}\left(\theta_{q}\right) \cos \left(M \phi_{q d}-2 \phi_{q}\right) d_{M 0}^{2}\left(\theta_{d}\right) \\
& =\sum_{M=-2}^{2} \widetilde{T}_{2 M}^{l}\left(\theta_{q}\right)\left[\cos \left(M \phi_{q d}\right) \cos \left(2 \phi_{q}\right)+\sin \left(M \phi_{q d}\right) \sin \left(2 \phi_{q}\right)\right] d_{M 0}^{2}\left(\theta_{d}\right), \\
A_{\gamma d}^{c V}\left(\theta_{q}, \phi_{q d}, \theta_{d}\right) & =\sum_{M=0}^{1} \widetilde{T}_{1 M}^{c}\left(\theta_{q}\right) \cos \left(M \phi_{q d}\right) d_{M 0}^{1}\left(\theta_{d}\right), \\
A_{\gamma d}^{c T}\left(\theta_{q}, \phi_{q d}, \theta_{d}\right) & =\sum_{M=1}^{2} \widetilde{T}_{2 M}^{c}\left(\theta_{q}\right) \sin \left(M \phi_{q d}\right) d_{M 0}^{2}\left(\theta_{d}\right) .
\end{aligned}
$$

Each of these generalized $A$-asymmetries are functions of $\theta_{q}$ and of some of the variables $\phi_{q}, \phi_{q d}$, and $\theta_{d}$. The first step is to isolate them by evaluating linear combinations of cross sections for different values of the appropriate polarization parameters $\left(P_{l, c}^{\gamma}, P_{1,2}^{d}\right)$. For example, $A_{\gamma}^{l}$ is obtained from the cross section difference for unpolarized deuterons and linearly polarized photons $\left(P_{l}^{\gamma}>0\right)$ minus the unpolarized one $\left(P_{l}^{\gamma}=0\right)$, i.e.

$$
A_{\gamma}^{l}=\frac{1}{P_{l}^{\gamma} S_{0}}\left[S\left(P_{l}^{\gamma}, 0,0,0\right)-S_{0}\right] .
$$

The others can be obtained by the following linear combinations of cross sections

$$
\begin{aligned}
A_{d}^{V}= & \frac{1}{2 P_{1}^{d} S_{0}}\left[S\left(0,0, P_{1}^{d}, P_{2}^{d}\right)-S\left(0,0,-P_{1}^{d}, P_{2}^{d}\right)\right] \\
A_{d}^{T}= & \frac{1}{2 P_{2}^{d} S_{0}}\left[S\left(0,0, P_{1}^{d}, P_{2}^{d}\right)+S\left(0,0,-P_{1}^{d}, P_{2}^{d}\right)-2 S_{0}\right], \\
A_{\gamma d}^{c V}= & \frac{1}{4 P_{c}^{\gamma} P_{1}^{d} S_{0}}\left[S\left(0, P_{c}^{\gamma}, P_{1}^{d}, P_{2}^{d}\right)-S\left(0,-P_{c}^{\gamma}, P_{1}^{d}, P_{2}^{d}\right)-S\left(0, P_{c}^{\gamma},-P_{1}^{d}, P_{2}^{d}\right)+S\left(0,-P_{c}^{\gamma},-P_{1}^{d}, P_{2}^{d}\right)\right], \\
A_{\gamma d}^{c T}= & \frac{1}{4 P_{c}^{\gamma} P_{2}^{d} S_{0}}\left[S\left(0, P_{c}^{\gamma}, P_{1}^{d}, P_{2}^{d}\right)-S\left(0,-P_{c}^{\gamma}, P_{1}^{d}, P_{2}^{d}\right)+S\left(0, P_{c}^{\gamma},-P_{1}^{d}, P_{2}^{d}\right)-S\left(0,-P_{c}^{\gamma},-P_{1}^{d}, P_{2}^{d}\right)\right], \\
A_{\gamma d}^{l V}= & \frac{1}{2 P_{l}^{\gamma} P_{1}^{d} S_{0}}\left[S\left(P_{l}^{\gamma}, 0, P_{1}^{d}, P_{2}^{d}\right)-S\left(0,0, P_{1}^{d}, P_{2}^{d}\right)-S\left(P_{l}^{\gamma}, 0,-P_{1}^{d}, P_{2}^{d}\right)+S\left(0,0,-P_{1}^{d}, P_{2}^{d}\right)\right], \\
A_{\gamma d}^{l T}= & \frac{1}{2 P_{l}^{\gamma} P_{2}^{d} S_{0}}\left[S\left(P_{l}^{\gamma}, 0, P_{1}^{d}, P_{2}^{d}\right)-S\left(0,0, P_{1}^{d}, P_{2}^{d}\right)+S\left(P_{l}^{\gamma}, 0,-P_{1}^{d}, P_{2}^{d}\right)-S\left(0,0,-P_{1}^{d}, P_{2}^{d}\right)\right. \\
& \left.-2 P_{l}^{\gamma} A_{\gamma}^{l} S_{0}\right] .
\end{aligned}
$$

Two of the generalized $A$-asymmetries in (A2) through (A8) yield directly one asymmetry each, namely $\widetilde{\Sigma}^{l}$ from $A_{\gamma}^{l}$ and $\widetilde{T}_{11}^{0}$ from $A_{d}^{V}$, i.e.

$$
\begin{aligned}
\widetilde{\Sigma}^{l} & =A_{\gamma}^{l}\left(\phi_{q}=0\right), \\
\widetilde{T}_{11}^{0} & =\sqrt{2} A_{d}^{V}\left(\phi_{q d}=\pi / 2, \theta_{d}=\pi / 2\right) .
\end{aligned}
$$

One could also obtain $\widetilde{\Sigma}^{l}$ from taking for linearly polarized photons the cross section difference of pions in the photon plane to pions perpendicular to this plane, i.e.

$$
\widetilde{\Sigma}^{l}=\frac{1}{P_{l}^{\gamma}} \frac{\left.S\left(P_{l}^{\gamma}>0,0,0,0\right)\right|_{\phi_{q}=0}-\left.S\left(P_{l}^{\gamma}>0,0,0,0\right)\right|_{\phi_{q}=\pi / 2}}{\left.S\left(P_{l}^{\gamma}>0,0,0,0\right)\right|_{\phi_{q}=0}+\left.S\left(P_{l}^{\gamma}>0,0,0,0\right)\right|_{\phi_{q}=\pi / 2}} .
$$


The remaining general asymmetries $A_{d}^{T}$ and $A_{\gamma d}^{(l / c)(V / T)}$ contain linear combinations of the asymmetries $\widetilde{T}_{2 M}^{0}$ and $\widetilde{T}_{I M}^{l / c}$. In order to separate the latter, one can exploit the dependence of the $A$-asymmetries on the angular variables $\phi_{q}, \phi_{q d}$, and $\theta_{d}$. This is achieved, following the analogous problem in deuteron electrodisintegration [44], by observing that the general functional form of an $A$-asymmetry is

$$
A^{I}\left(\phi_{q}, \phi_{q d}, \theta_{d}\right)=\sum_{M=-I}^{I} \alpha_{I M}\left(\phi_{q}, \phi_{q d}\right) d_{M 0}^{I}\left(\theta_{d}\right), \quad(I=1,2),
$$

where

$$
\alpha_{I M}\left(\phi_{q}, \phi_{q d}\right)=c_{I M}\left(\phi_{q}\right) \cos M \phi_{q d}+s_{I M}\left(\phi_{q}\right) \sin M \phi_{q d},
$$

and the $\phi_{q}$-dependent functions $c_{I M}\left(\phi_{q}\right)$ and $s_{I M}\left(\phi_{q}\right)$ have either the form

$$
a_{0}+a_{1} \cos \phi_{q}+a_{2} \cos 2 \phi_{q}
$$

or

$$
b_{1} \sin \phi_{q}+b_{2} \sin 2 \phi_{q} .
$$

One should note, that for $A_{\gamma d}^{c(V / T)}$ the $\phi_{q}$-dependence is absent, i.e. $c_{I M} \equiv 0$ or $s_{I M} \equiv 0$, and the sum over $M$ in (A19) runs from 0 through $I$. For a given $I$ the $M$-components $\alpha_{I M}\left(\phi_{q}, \phi_{q d}\right)$ of the asymmetry $A^{I}\left(\phi_{q}, \phi_{q d}, \theta_{d}\right)$ can be separated by a proper choice of $\theta_{d}$ exploiting the properties of the small $d_{M 0}^{I}$-functions. For $I=1$ (vector asymmetries), taking $\theta_{d}=0$ or $\pi / 2$, i.e. $d_{M 0}^{1}(0)=\delta_{M 0}$ or $d_{M 0}^{1}(\pi / 2)=M / \sqrt{2}$, yields $\alpha_{10}$ or $\alpha_{11}$, respectively, and for the tensor asymmetries $(I=2)$ one may first choose $\theta_{d}=0$ yielding with $d_{M 0}^{2}(0)=\delta_{M 0}$ directly $\alpha_{20}$. The latter being determined, then setting $\theta_{d}=\pi / 4$ and $\pi / 2$, one can obtain the remaining two terms $\alpha_{21}$ and $\alpha_{22}$. For the separation of $\alpha_{21}$ and $\alpha_{22}$ one can also choose $\theta_{d}=\theta_{d}^{0}=\operatorname{arcos}(1 / \sqrt{3})$ together with $\phi_{q d}$ and $\phi_{q d}+\pi$. Then the sum and difference of the corresponding asymmetries result in $\alpha_{21}$ and $\alpha_{22}$, respectively.

In the next step, in order to separate the two contributions $c_{I M}$ and $s_{I M}$ in A20 , one can take first $\phi_{q d}=0$ giving $c_{I M}$ and then $\phi_{q d}=\pi /(2 M)$ for $M \neq 0$ which yields directly $s_{I M}$. The remaining separation of the coefficients $a_{n}$ or $b_{n}$ in (A21) is then achieved by appropriate choices of $\phi_{q}$. This completes the separation.

\section{APPENDIX B: A MODIFIED IMPULSE APPROXIMATION}

For incoherent $\pi^{0}$-photoproduction in IA, the $N N$-final state is described by a plane wave $\left|\vec{p}, s m_{s}\right\rangle$ which is not orthogonal to the deuteron wave function. Thus the IA matrix element contains a spurious contribution from coherent $\pi^{0}$-photoproduction, the size of which is governed by the overlap between the plane wave and the deuteron wave function which is just the deuteron wave function in momentum space (see Eq. (6))

$$
\phi_{m_{s} m_{d}}(\vec{p})=\left\langle\vec{p}, 1 m_{s} \mid 1 m_{d}\right\rangle^{(d)} \neq 0,
$$

whereas, if the interaction between the nucleons is properly taken into account, the overlap with the final $N N$ scattering wave vanishes. Therefore, one can expect that a large fraction of $N N$-rescattering effects in incoherent $\pi^{0}$-photoproduction arises from the elimination of the spurious coherent contribution.

This spurious contribution can be avoided by applying a modified IA, in which one uses a modified $N N$-final state wave function, where the deuteron wave function component is projected out by the replacement

$$
\left|\vec{p}, 1 m_{s}\right\rangle \rightarrow\left|\vec{p}, 1 m_{s}\right\rangle-\sum_{m_{d}}\left|1 m_{d}\right\rangle^{(d)}\left\langle 1 m_{d} \mid \vec{p}, 1 m_{s}\right\rangle=\left|\vec{p}, 1 m_{s}\right\rangle-\sum_{m_{d}}\left|1 m_{d}\right\rangle^{(d)} \phi_{m_{s} m_{d}}^{*}(\vec{p}) \text {. }
$$

This means the following replacement for the IA matrix element

$$
\left\langle\vec{p}, 1 m_{s}|T| 1 m_{d}\right\rangle^{(d)} \rightarrow\left\langle\vec{p}, 1 m_{s}|T| 1 m_{d}\right\rangle^{(d)}-\sum_{m_{d}^{\prime}} \phi_{m_{s} m_{d}^{\prime}}(\vec{p})^{(d)}\left\langle 1 m_{d}^{\prime}|T| 1 m_{d}\right\rangle^{(d)}
$$

The matrix element ${ }^{(d)}\left\langle 1 m_{d}^{\prime}|T| 1 m_{d}\right\rangle^{(d)}$ corresponds to the one for coherent pion photoproduction $\gamma d \rightarrow \pi^{0} d$ in the off-shell region. The comparison of the original IA with the modified IA reveals then what fraction of the whole FSI-effect arises from the non-orthogonality.

[1] G.F. Chew and H.W. Lewis, Phys. Rev. 84, 779 (1951). 
[2] M. Lax and H. Feshbach, Phys. Rev. 88, 509 (1952).

[3] I. Blomqvist and J.M. Laget, Nucl. Phys. A 280, 405 (1977).

[4] J.M. Laget, Nucl. Phys. A 296, 388 (1978).

[5] J.M. Laget, Phys. Rep. 69, 1 (1981).

[6] P. Benz et al., Nucl. Phys. B 65, 158 (1973).

[7] M.I. Levchuk, V.A. Petrun'kin, and M. Schumacher, Z. Phys. A 355, 317 (1996).

[8] M.I. Levchuk, M. Schumacher, and F. Wissmann, nucl-th/0011041

[9] R.A. Arndt et al., SAID: http://gwdac.phys.gwu.edu/

[10] D. Drechsel, O. Hanstein, S.S. Kamalov, and L. Tiator, MAID: http://www.kph.uni-mainz.de/de/MAID/maid2000/

[11] R. Machleidt, K. Holinde, and Ch. Elster, Phys. Rep. 149, 1 (1987); R. Machleidt, Adv. Nucl. Phys. 19, 189 (1989).

[12] M.I. Levchuk, M. Schumacher, and F. Wissmann, Nucl. Phys. A 675, 621 (2000).

[13] A. Loginov, A.Sidorov, and V. Stibunov, Phys. Atom. Nucl. 63,391 (2000) (Yad. Fiz. 63, 459 (2000)).

[14] E.M. Darwish, H. Arenhövel, and M. Schwamb, Eur. Phys. J. A 16, 111 (2003).

[15] E.M. Darwish, H. Arenhövel, and M. Schwamb, Eur. Phys. J. A 17, 513 (2003).

[16] Proc. Second Int. Symposium on the GDH sum rule and the Spin Structure of the Nucleon, Genova 2002, eds. M. Anghinolfi, M. Battaglieri, and R. de Vita (World Scientific, Singapore 2003); Proc. Third Int. Symposium on the GDH Sum Rule and its Extensions, Norfolk, Virginia, 2004, eds. S. Kuhn and J.-P. Chen, (World Scientific, Singapore 2005).

[17] R. Schmidt, H. Arenhövel, and P. Wilhelm, Z. Phys. A 355, 421 (1996).

[18] E.M. Darwish, J. Phys. G 31, 105 (2005).

[19] E.M. Darwish, Nucl. Phys. A 735, 200 (2005).

[20] E.M. Darwish, Nucl. Phys. A 748, 596 (2005).

[21] E.M. Darwish, nucl-th/0504031

[22] E.M. Darwish and A. Salam, nucl-th/0505002

[23] H. Arenhövel and A. Fix, to be published.

[24] H. Arenhövel, A. Fix, and M. Schwamb, Phys. Rev. Lett. 93, 202301 (2004).

[25] A. Fix and H. Arenhövel, Z. Phys. A 359, 427 (1997).

[26] J. Haidenbauer and W. Plessas, Phys. Rev. C 30, 1822 (1984); 32, 1424 (1985).

[27] S. Nozawa, B. Blankleider, and T.-S.H. Lee, Nucl. Phys. A 513, 459 (1990).

[28] G.F. Chew, M.L. Goldberger, F.E. Low, and Y. Nambu, Phys. Rev. 106, 359 (1957).

[29] A. Salam and H. Arenhövel, Phys. Rev. C 70, 044008 (2004).

[30] J. Noble, Phys. Lett. B 67, 39 (1977).

[31] U. Siodlaczek et al., Eur. Phys. J. A 10,365 (2001).

[32] M. Asai et al., Phys. Rev. C 42, 837 (1990).

[33] B. Krusche et al., Eur. Phys. J. A 6, 309 (1999).

[34] C. Reiss, H. Arenhövel, and M. Schwamb, nucl-th/0505030

[35] E. C. Booth, B. Chasan, J. Comuzzi, and P. Bosted, Phys. Rev. C 20, 1217 (1979).

[36] V. Lensky, V. Baru, J. Haidenbauer, C. Hanhart, A.E. Kudryavtsev, and U.-G. Meissner, nucl-th/0505039

[37] G. Köbschall, B. Alberti, H. Jansen, K. Rohrich, C. Schmitt, V.H. Walther, K. Weinand, M. Kobayashi, and H. Arenhövel, Nucl. Phys. A 466, 612 (1987).

[38] L. Levchuk, L. Canton, and A. Shebeko, Eur. Phys. J. A 21, 29 (2004), nucl-th/0311004.

[39] A. Sandorfi, private communication.

[40] T.-S. H. Lee, private communication.

[41] P. Pedroni, private communication.

[42] C. Bacci et al., Phys. Lett. B 39, 559 (1972).

[43] Y. Hemmi et al., Nucl. Phys. B 55, 333 (1975).

[44] H. Arenhövel, W. Leidemann, and E.L. Tomusiak, Eur. Phys. J. A 23, 147 (2005). 

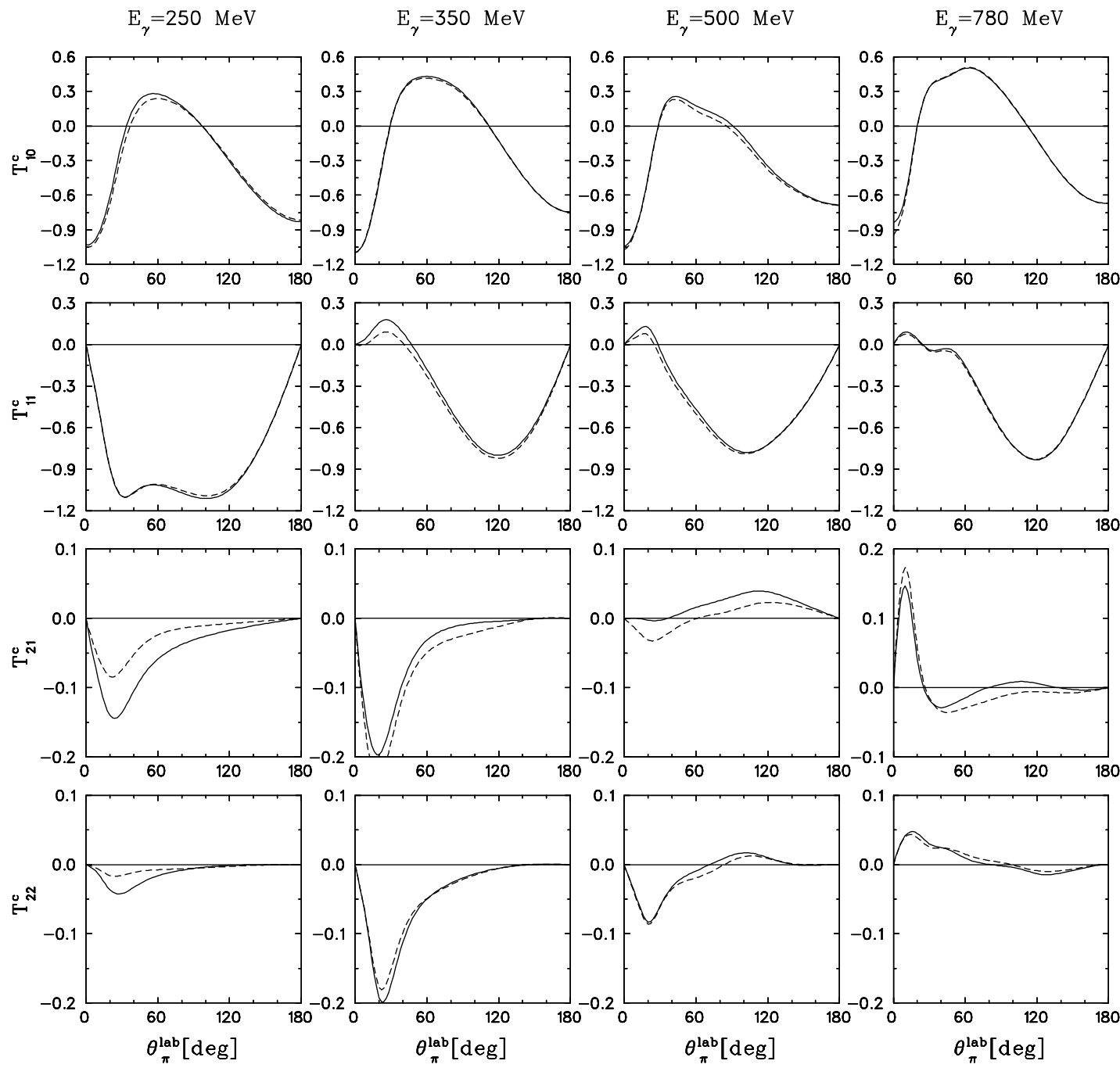

FIG. 12: Beam-target asymmetries $T_{I M}^{c}$ for circularly polarized photons and polarized deuterons for semi-exclusive $\pi^{-}$photoproduction on the deuteron. Notation of curves as in Fig. 8 

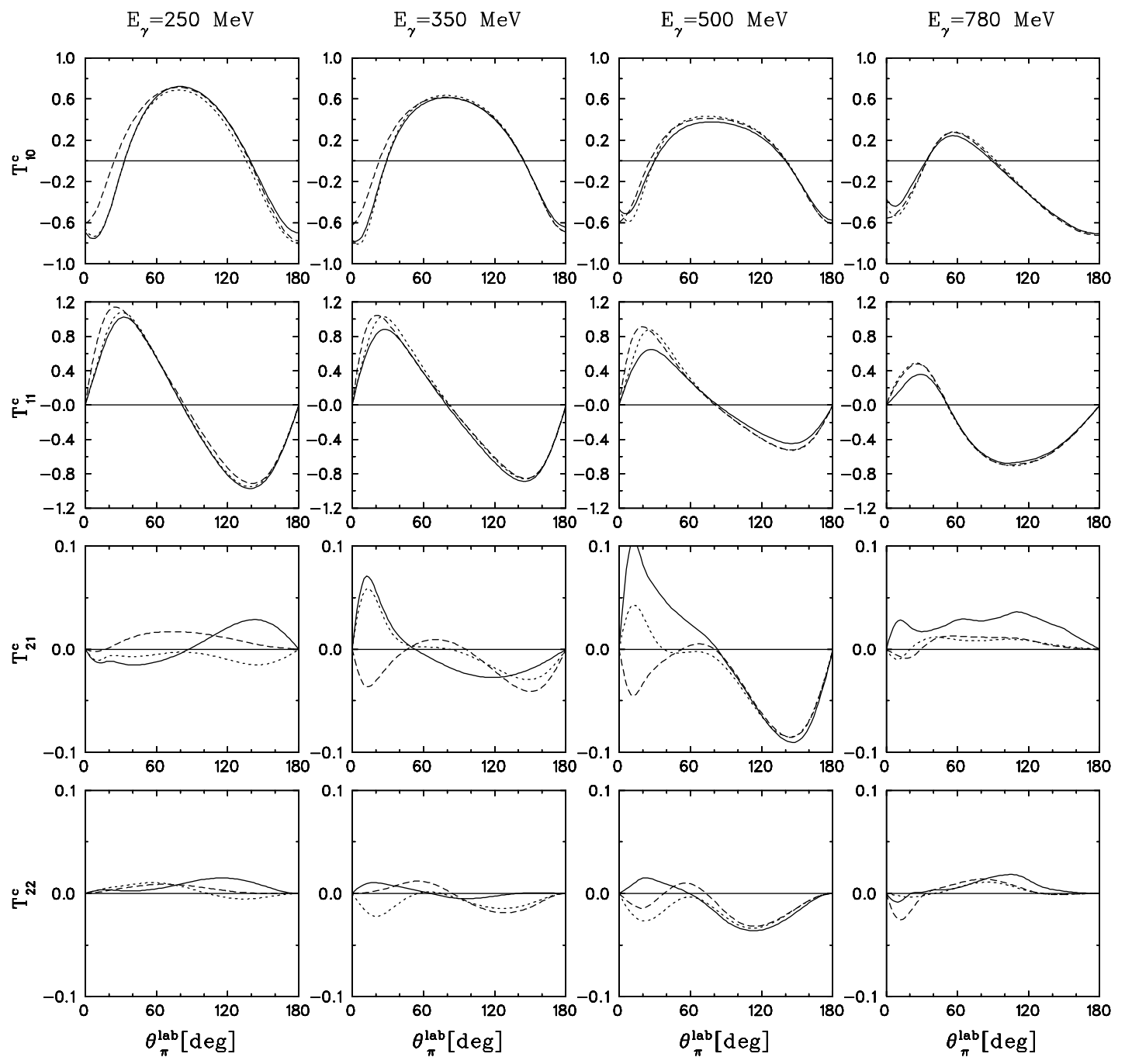

FIG. 13: Beam-target asymmetries $T_{I M}^{c}$ for circularly polarized photons and polarized deuterons for semi-exclusive $\pi^{0}-$ photoproduction on the deuteron. Notation of curves as in Fig. 8 

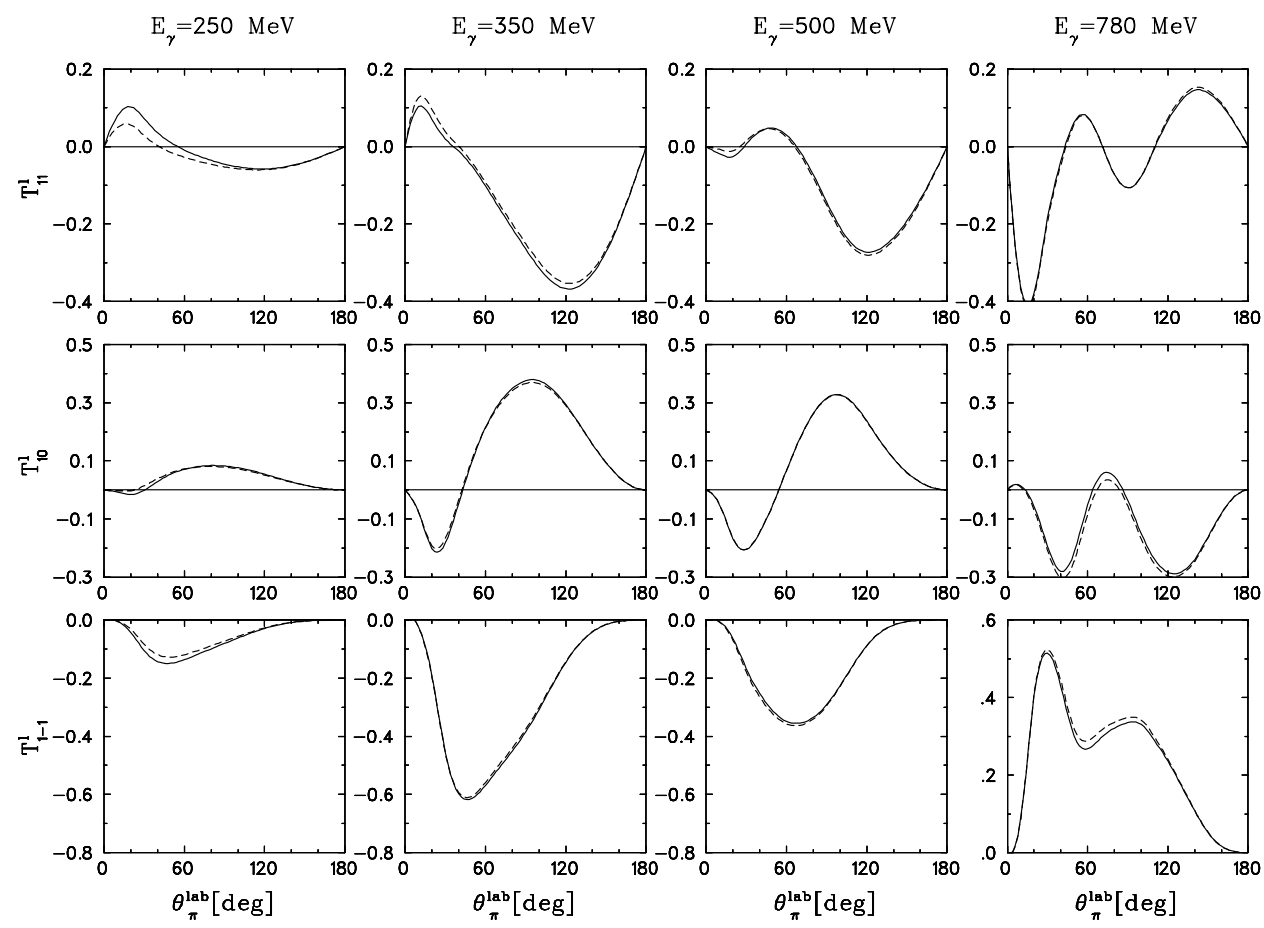

FIG. 14: Beam-target asymmetries $T_{1 M}^{l}$ for linearly polarized photons and vector polarized deuterons for semi-exclusive $\pi^{-}-$ photoproduction on the deuteron. Notation of curves as in Fig. 8
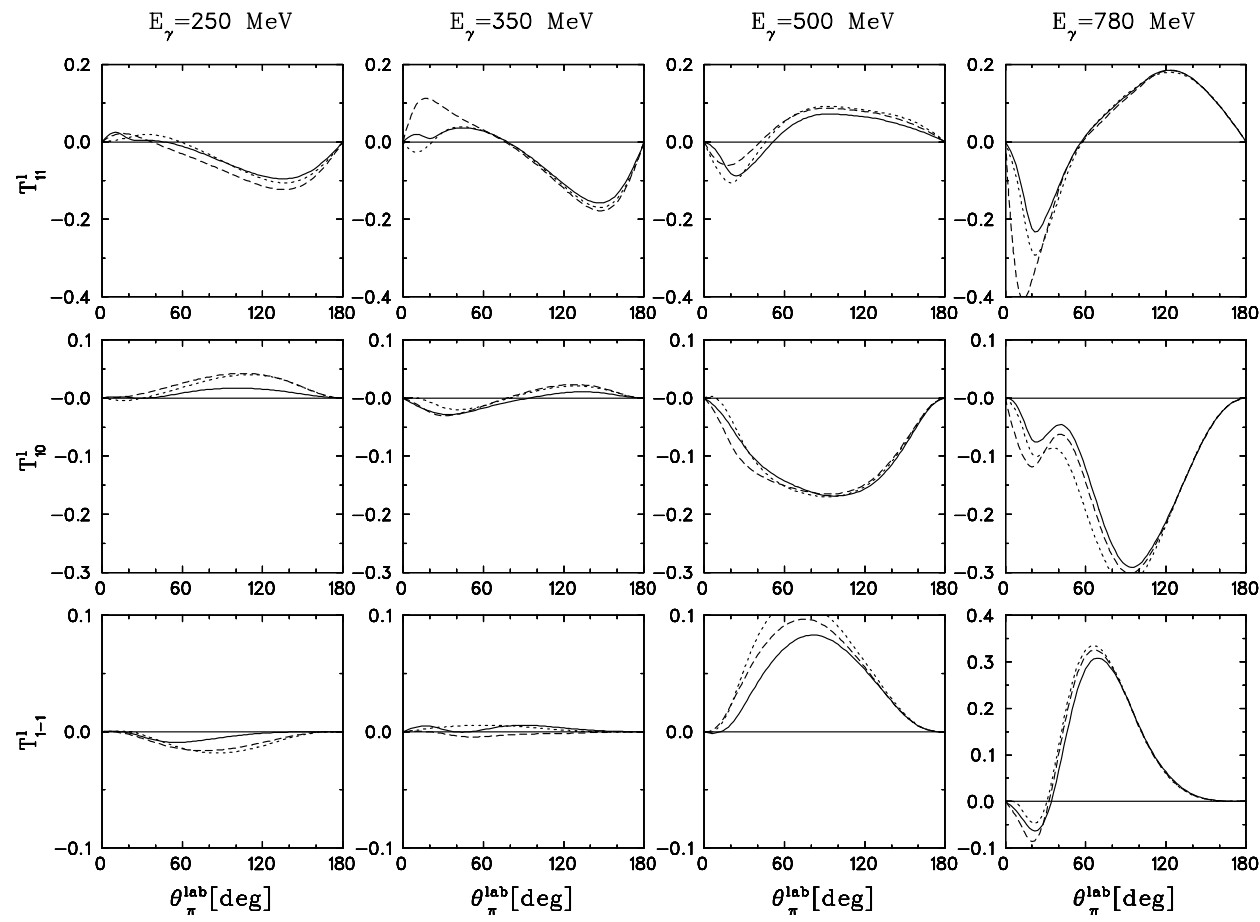

FIG. 15: Beam-target asymmetries $T_{1 M}^{l}$ for linearly polarized photons and vector polarized deuterons for semi-exclusive $\pi^{0}$ photoproduction on the deuteron. Notation of curves as in Fig. 8 


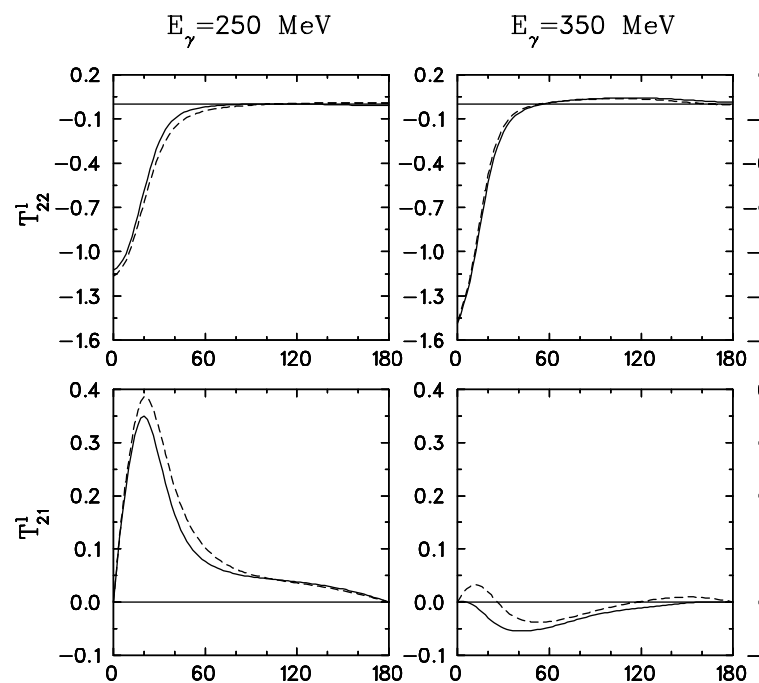

$\mathrm{E}_{\gamma}=500 \mathrm{MeV}$
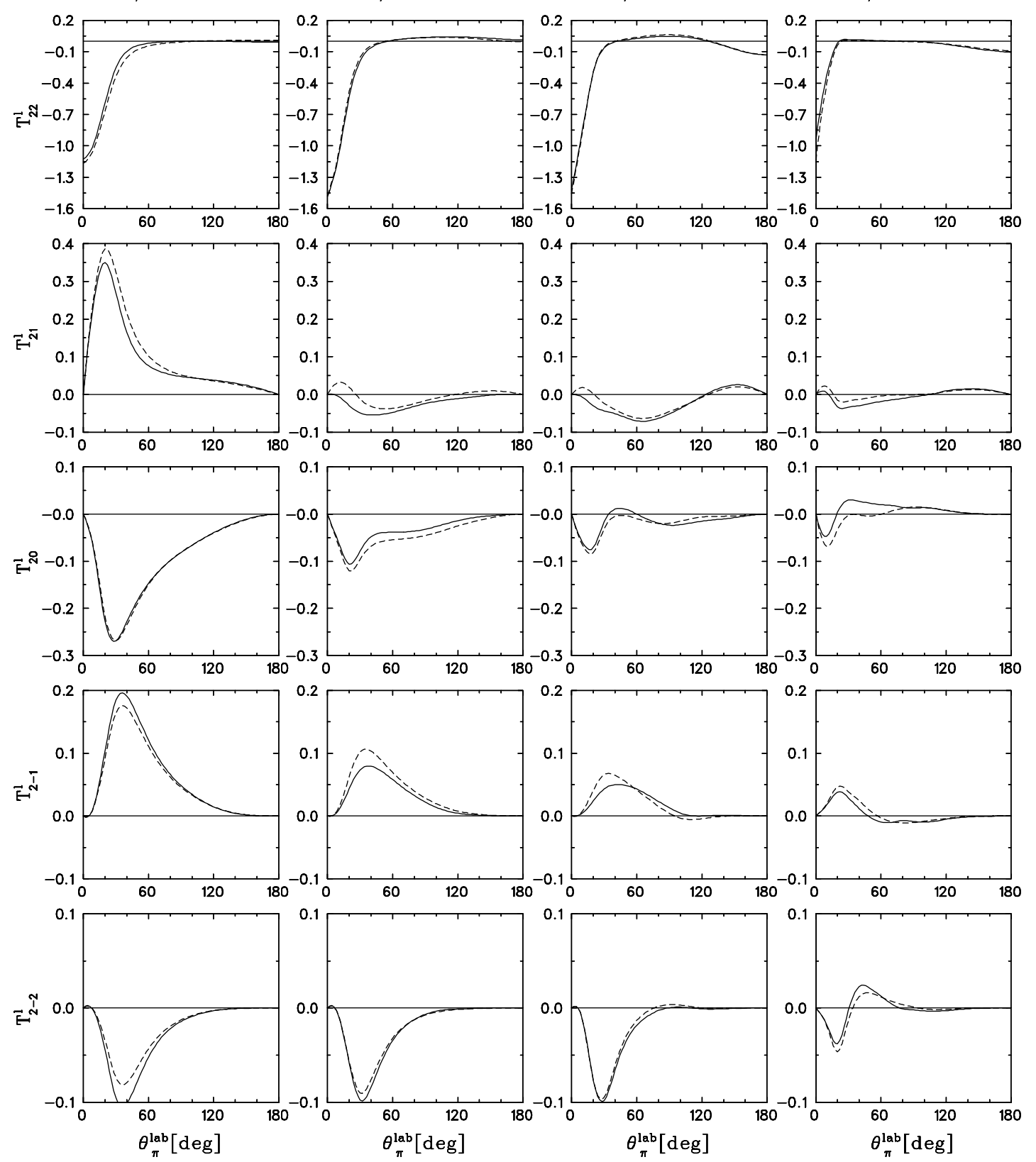

FIG. 16: Beam-target asymmetries $T_{2 M}^{l}$ for linearly polarized photons and tensor polarized deuterons for semi-exclusive $\pi^{-}$photoproduction on the deuteron. Notation of curves as in Fig. 8 


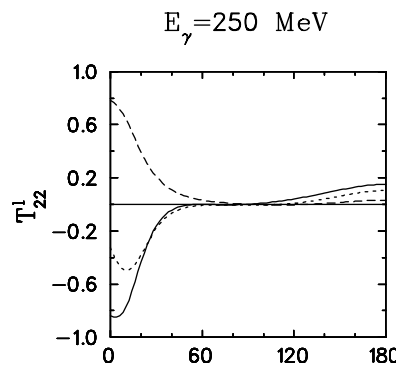

$\mathrm{E}_{\gamma}=350 \mathrm{MeV}$

$\mathrm{E}_{\gamma}=500 \mathrm{MeV}$

$\mathrm{E}_{\gamma}=780 \mathrm{MeV}$
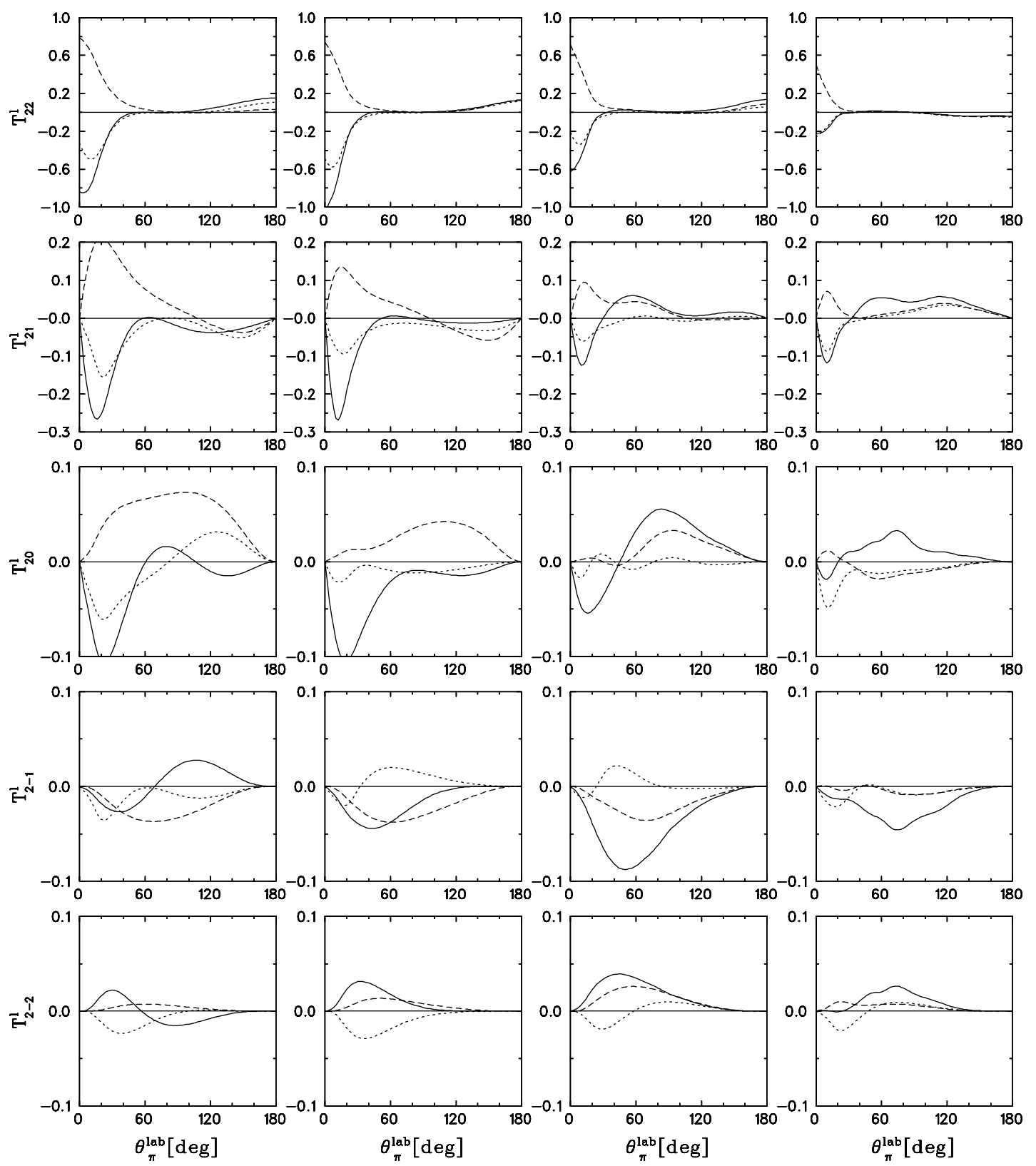

FIG. 17: Beam-target asymmetries $T_{2 M}^{l}$ for linearly polarized photons and tensor polarized deuterons for semi-exclusive $\pi^{0}$ photoproduction on the deuteron. Notation of curves as in Fig. 8 

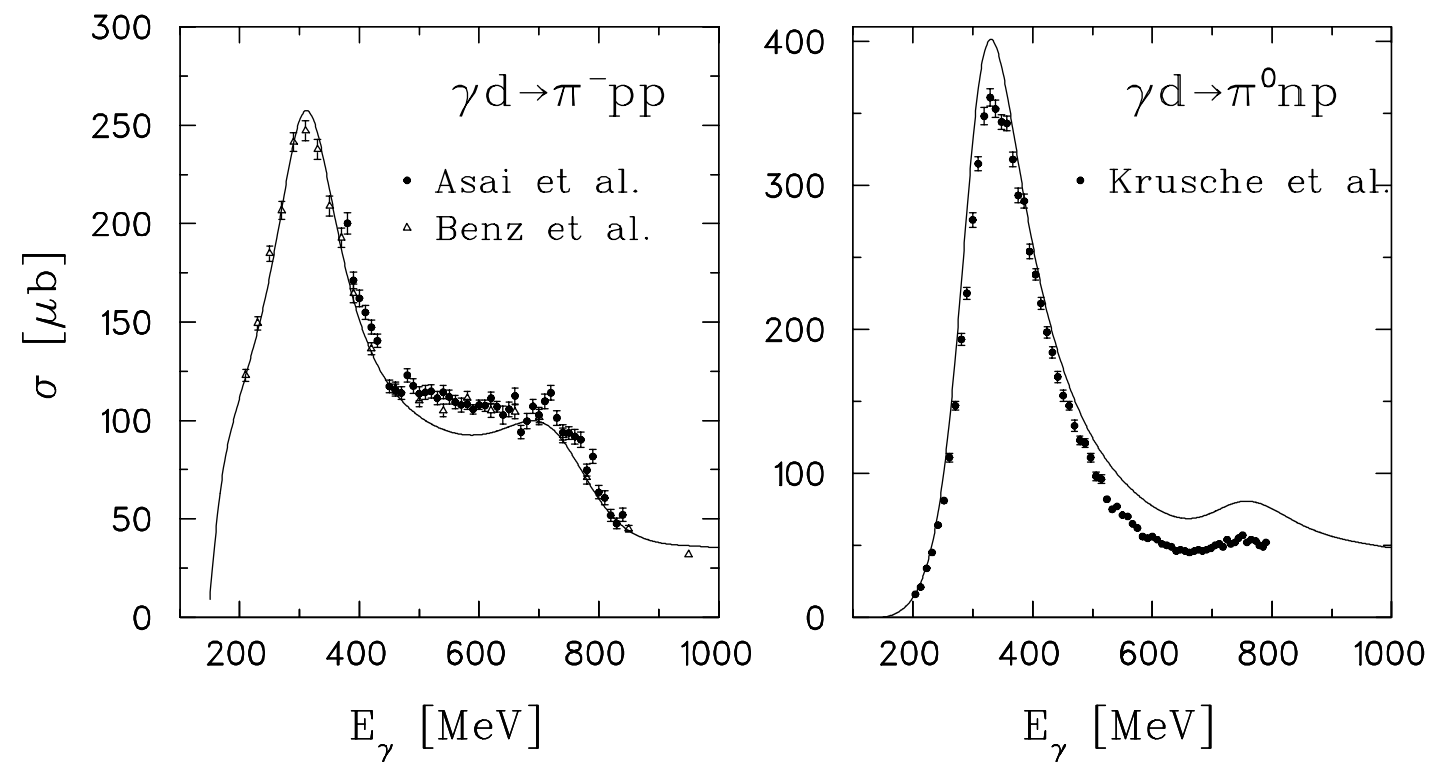

FIG. 18: Total cross section for $\pi^{-}$(left panel) and $\pi^{0}$-photoproduction (right panel) on the deuteron. Solid curves: IA $+N N-$ and $\pi N$-rescattering. Experimental data from Benz et al. [6] and from Asai et al. [32] for $\pi^{-}$- and from Krusche et al. 33] for $\pi^{0}$-production.
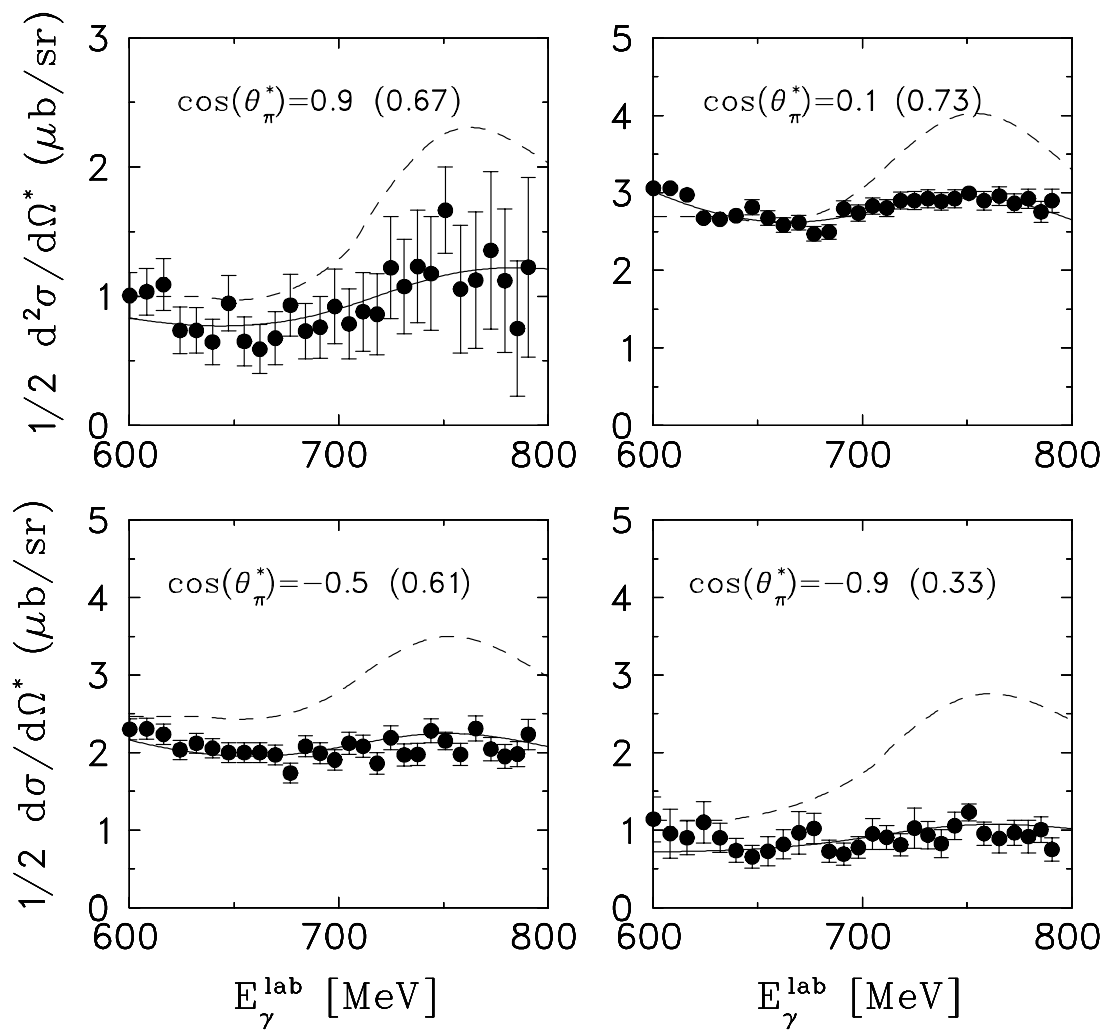

FIG. 19: Differential cross section for $\pi^{0}$-production on the deuteron as function of the lab photon energy for fixed pion angles in the equivalent $\gamma N$ c.m. system. The cross section is plotted per nucleon. Notation of curves: solid: full calculation multiplied by the factors in parentheses; dashed: MAID prediction for the sum $1 / 2\left(d \sigma\left(\pi^{0} p\right)+d \sigma\left(\pi^{0} n\right)\right)$. 


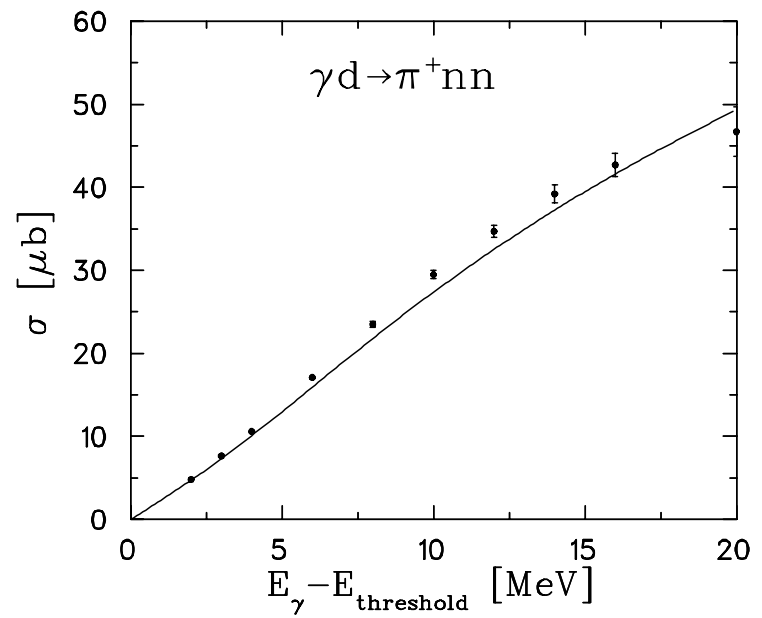

FIG. 20: Total cross section near threshold for $\pi^{+}$-photoproduction on the deuteron. Solid curve: IA $+N N-$ and $\pi N-$ rescattering. Experimental data from Booth et al. [35].
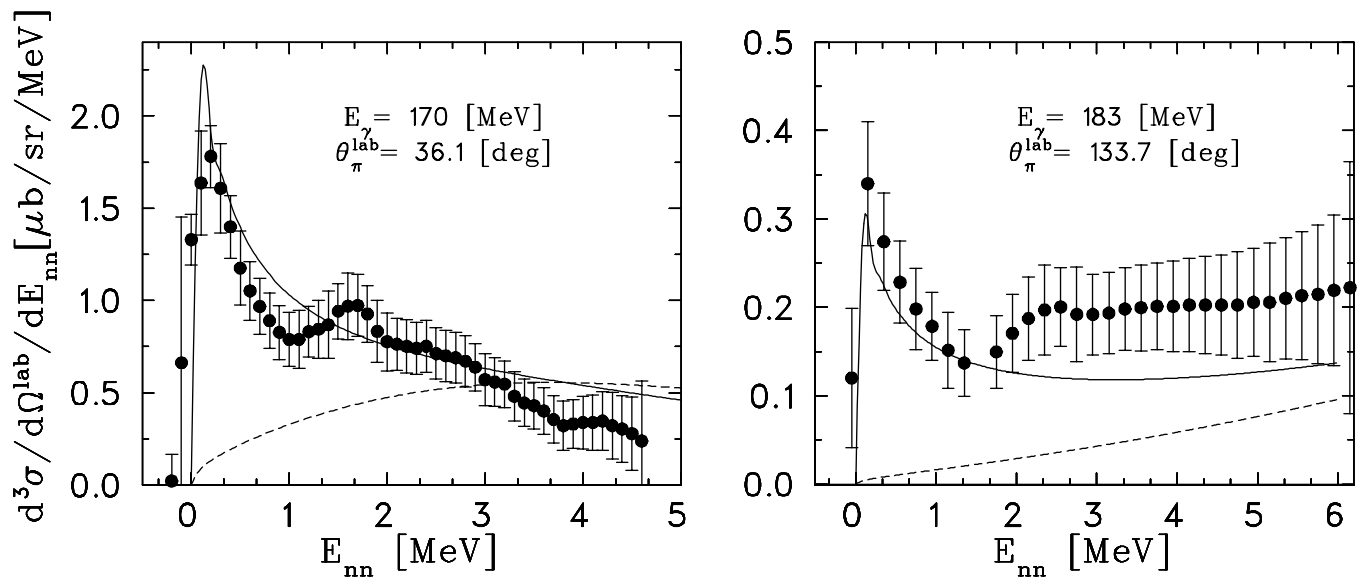

FIG. 21: Semi-exclusive differential cross section $d^{3} \sigma / d \Omega_{\pi} d E_{n n}$ for $\pi^{+}$-photoproduction on the deuteron at fixed pion angle as function of the relative energy $E_{n n}$ of the final two neutrons. Solid curves: IA $+N N$-rescattering. Experimental data from Köbschall et al. [37]. 

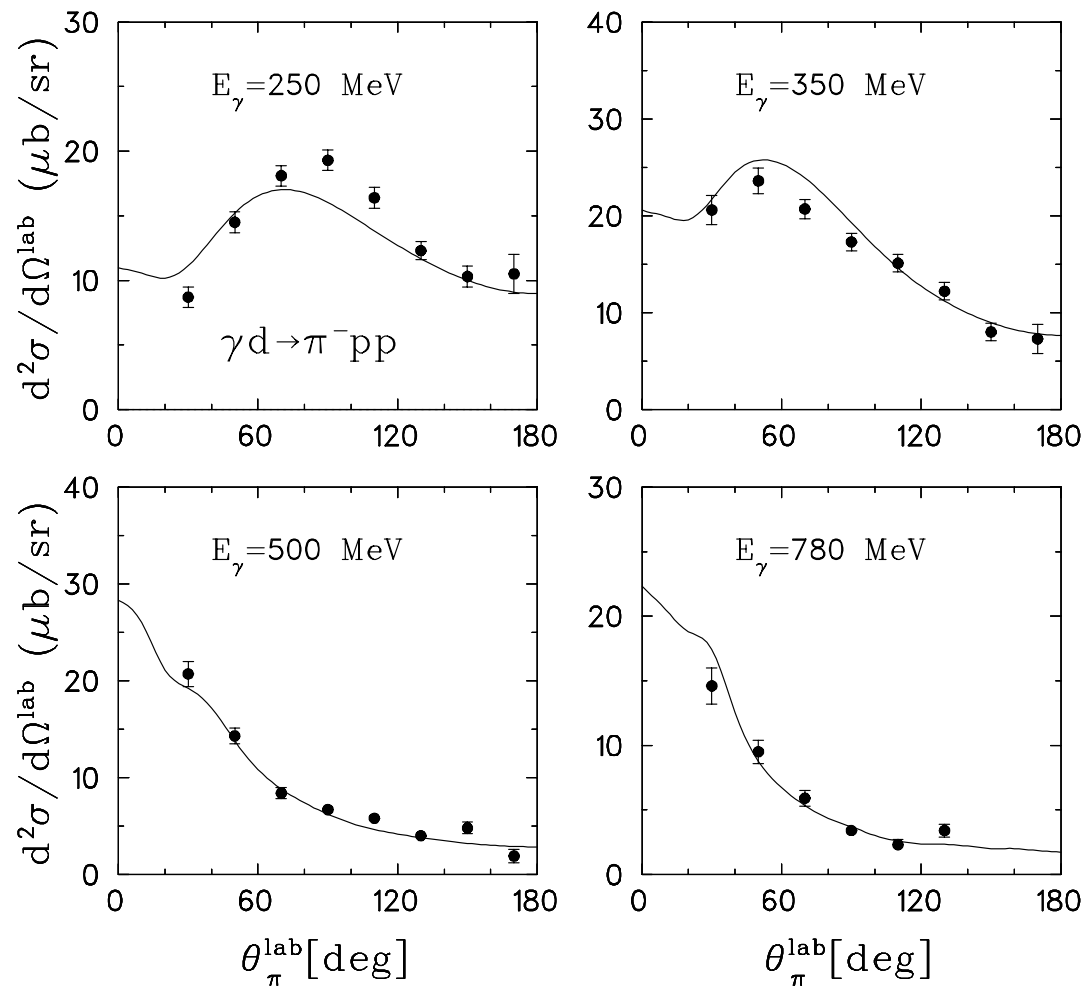

FIG. 22: Semi-exclusive differential cross section for $\pi^{-}$-photoproduction on the deuteron. Solid curves: IA $+N N$-rescattering. Experimental data from Benz et al. [6].
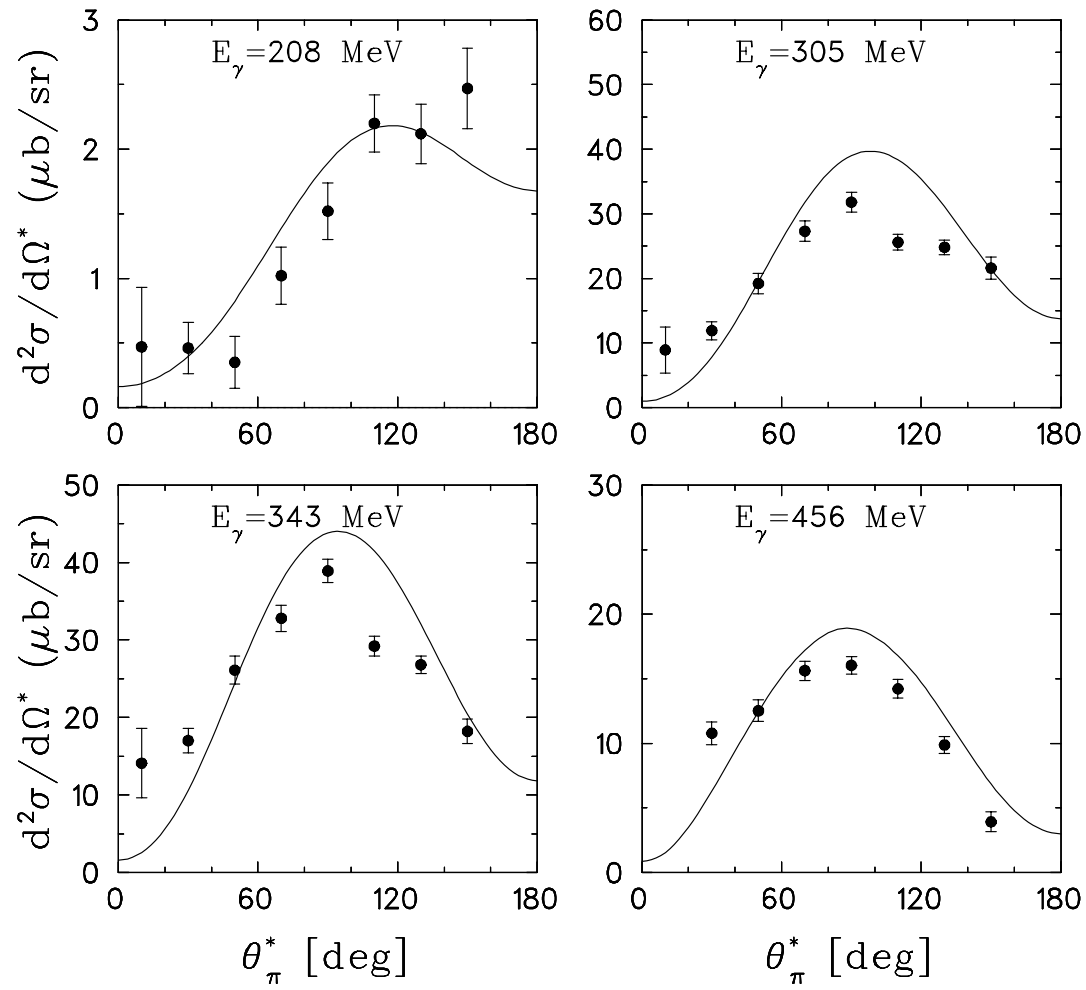

FIG. 23: Semi-exclusive differential cross section for $\pi^{-}$-photoproduction on the deuteron. Cross section and the pion angle $\theta_{\pi}^{*}$ refer to the equivalent $\gamma N$ c.m. system of the corresponding reaction on the nucleon. Solid curves: IA $+N N$-rescattering. Experimental data from Krusche et al. 33]. 

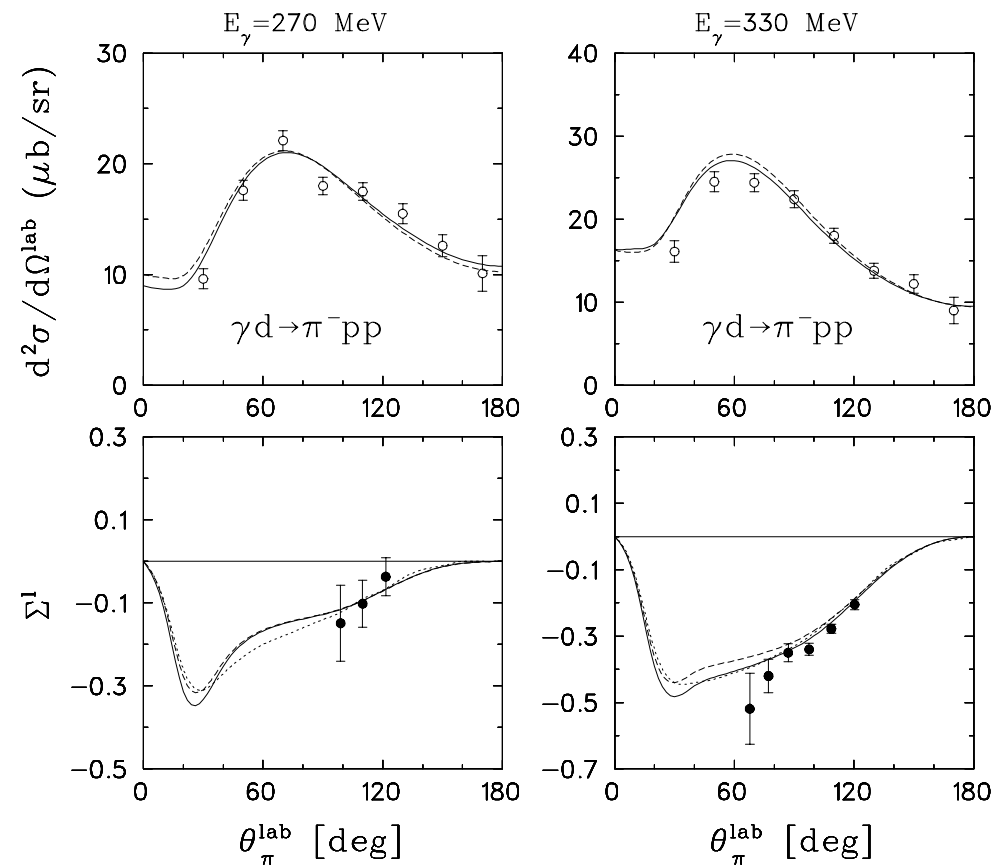

FIG. 24: Differential cross section and linear photon polarization asymmetry for semi-exclusive $\pi^{-}$photoproduction on the deuteron. Notation of curves: dashed: IA; dotted: IA from Lee and Sato 40]; solid: IA $+N N$-rescattering. The data are for the differential cross section from Benz et al [6] and for the linear photon asymmetry from the LEGS-collaboration (LEGS-exp.L3b) [39].

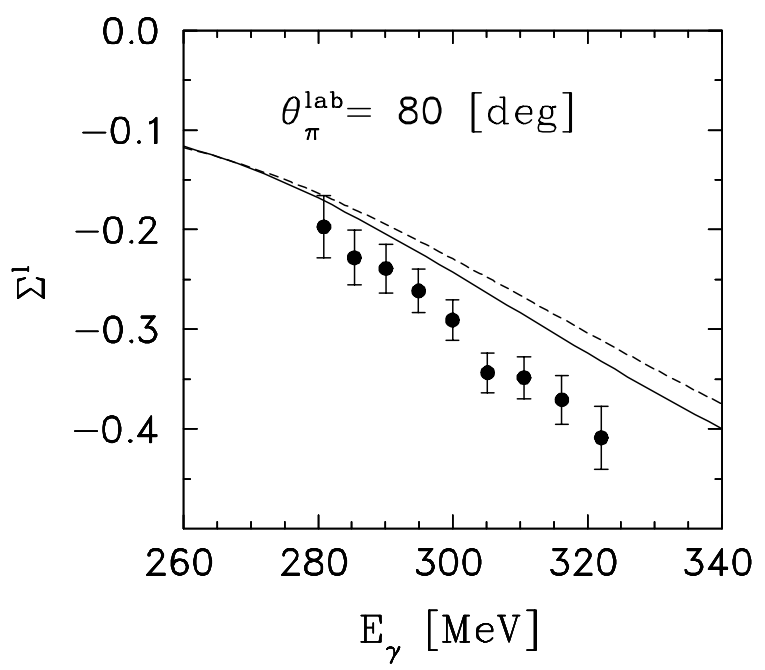

FIG. 25: Linear photon polarization asymmetry for semi-exclusive $\pi^{-}$-photoproduction on the deuteron at constant pion emission angle $\theta_{\pi}=80^{\circ}$ as function of photon energy. Notation of curves: dashed: IA; solid: IA $+N N-$ rescattering. The data are from the LEGS-collaboration (LEGS-exp.L3b) [39]. 

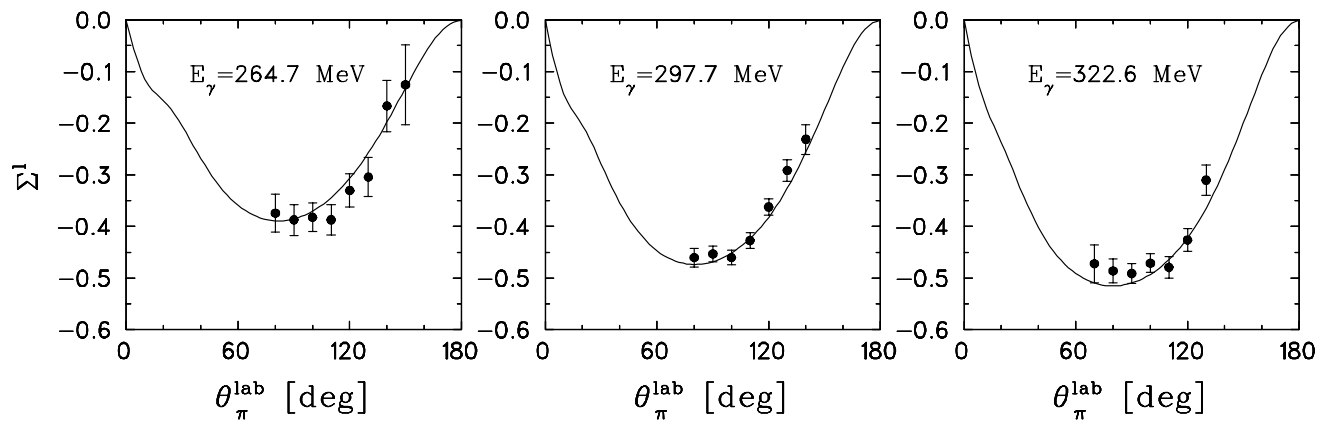

FIG. 26: Linear photon polarization asymmetry for semi-exclusive $\pi^{0}$-photoproduction on the deuteron. Solid curves: IA + $N N$-rescattering. Experimental data from the LEGS-collaboration (LEGS-exp.L3b) [39].
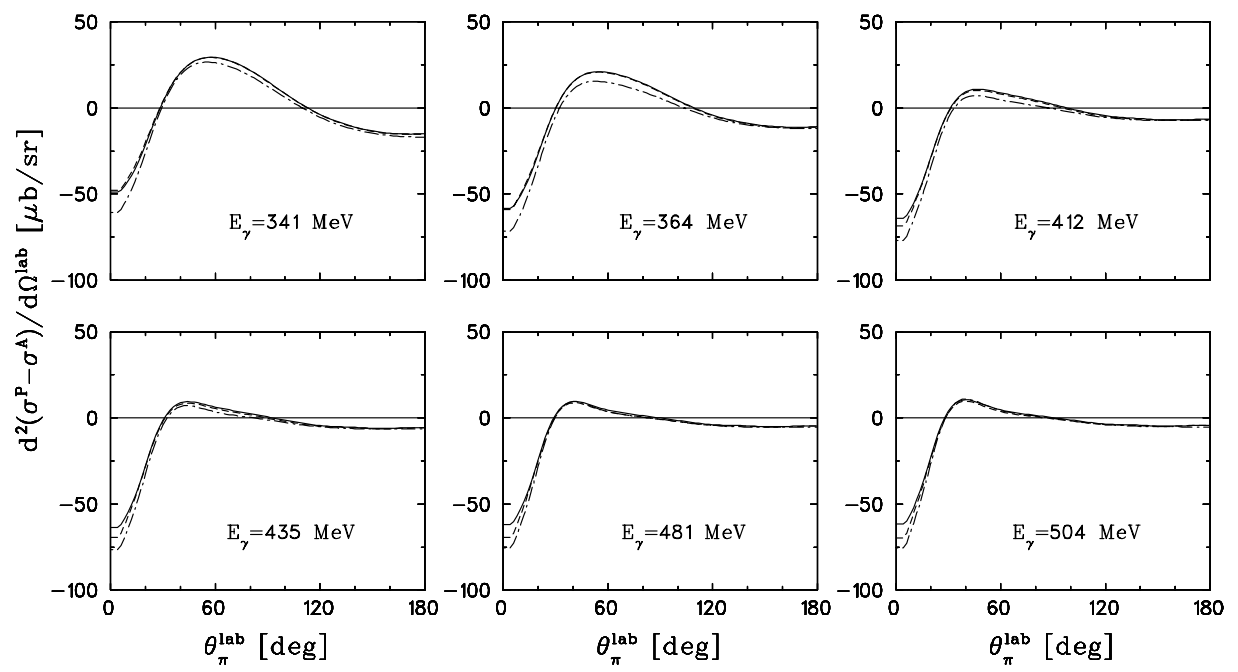

FIG. 27: Circular photon polarization asymmetry for semi-exclusive $\pi^{-}$-photoproduction on the deuteron. Notation of curves: dashed: IA; solid: IA + NN-rescattering; dash-dot: corresponding elementary cross section. 